\title{
Double-sided Parisian option pricing
}

\author{
J.H.M. Anderluh · J.A.M. van der Weide
}

Received: 8 June 2007 / Accepted: 13 November 2008 / Published online: 30 January 2009

(C) The Author(s) 2009. This article is published with open access at Springerlink.com

\begin{abstract}
In this paper we derive Fourier transforms for double-sided Parisian option contracts. The double-sided Parisian option contract is triggered by the stock price process spending some time above an upper level or below some lower level. The double-sided Parisian knock-in call contract is the general type of Parisian contract from which also the single-sided contract types follow. The paper gives an overview of the different types of contracts that can be derived from the double-sided Parisian knock-in calls, and, after discussing the Fourier inversion, it concludes with various numerical examples, explaining the, sometimes peculiar, behavior of the Parisian option.

The paper also yields a nice result on standard Brownian motion. The Fourier transform for the double-sided Parisian option is derived from the Laplace transform of the double-sided Parisian stopping time. The probability that a standard Brownian motion makes an excursion of a given length above zero before it makes an excursion of another length below zero follows from this Laplace transform and is not very well known in the literature. In order to arrive at the Laplace transform, a very careful application of the strong Markov property is needed, together with a non-intuitive lemma that gives a bound on the value of Brownian motion in the excursion.
\end{abstract}

Keywords Parisian options · Excursions · Fourier inversion

Mathematics Subject Classification (2000) $60 \mathrm{G} 40 \cdot 62 \mathrm{~L} 15 \cdot 60 \mathrm{~J} 65$

JEL Classification $\mathrm{G} 12 \cdot \mathrm{G} 13$

J.H.M. Anderluh $(\bowtie) \cdot$ J.A.M. van der Weide

TUDelft, Delft Institute of Applied Mathematics, P.O. Box 5031, 2600 GA Delft, The Netherlands e-mail: J.H.M.Anderluh@TUDelft.nl 


\section{Introduction}

The Parisian option is a kind of barrier option with the difference that the contract is not specified in terms of touching a barrier, but in terms of staying above or below the barrier for a certain period of time. The interest in these options is motivated by the study of structured products, insurance and investment problems. Convertible bonds and problems in real options contain Parisian optionality; the Parisian option contract itself is at the present time not exchange-traded. Details about the practical differences between standard barrier options and Parisian options are discussed in [8], the first paper on Parisian options. The way Parisian options turn up in real option problems is treated in [11], for an application in the direction of convertible bonds see [18]. For applications in credit risk and life insurance see [19] and [6] respectively. The authors in [8] derived Laplace transforms for the single-sided version, which is extended in [11] to a Parisian type of contract that is triggered by staying a period of time above the barrier or hitting a level exceeding this barrier. Chesney and Gauthier treat American Parisian options in [7]. Here we treat the pricing of the double-sided Parisian option and, like the papers previously mentioned, we use Fourier (or Laplace) transforms to achieve this. The calculation of Fourier transforms instead of Laplace transforms is motivated by the fact that a lot of numerical Laplace inversion algorithms are using the complex continuation of Laplace transforms to Fourier transforms for the actual inversion; see e.g. [14]. As we want to conclude our paper by a section on numerical examples, we have to invert the Fourier transforms we calculate. In [13] the authors use a PDE method approach to solve the Parisian option pricing problem, but convergence turns out to be rather slow, which is a result of the local behavior of Brownian motion.

The reason to treat double-sided Parisian options, apart from that there may be practical applications to this type of optionality, is that this contract type is rather general. After analyzing the double-sided Parisian knock-in call contract, we are able to give prices for the single-sided versions as well. We do not need to derive separate formulas for Parisian down-and-out calls, Parisian up-and-in puts, and so on. Prices for all of these contract types can be computed from the Fourier transform of the doublesided Parisian knock-in call. The concluding numerical examples will show the reader how the various Parisian option types behave that can be constructed from the doublesided Parisian knock-in call. The double-sided Parisian option treated by [17] differs from the one treated here as will be pointed out in the next section.

In order to arrive at the Fourier transform for the double-sided Parisian option, the Laplace transform of the double-sided Parisian stopping time is calculated. This calculation is rather cumbersome in the sense that it needs a very careful application of the strong Markov property. Moreover, an application of the optional sampling theorem has to be justified by proving that Brownian motion, stopped at the double-sided Parisian stopping time, is uniformly integrable. Finally, from this Laplace transform follows the probability that Brownian motion makes an excursion of length $D_{1}$ below zero before it makes an excursion of length $D_{2}$ above zero as a remarkably simple formula that, as far as the authors know, is not well known in the literature.

The paper is organized as follows. In Sect. 2 we introduce the double-sided Parisian option and the relevant notation. In order to price the contract, we rewrite 
the pricing problem into the problem of calculating a probability. In Sect. 3 we derive Laplace transforms for the double-sided Parisian stopping time and the value of standard Brownian motion at that stopping time. Section 4 treats the actual Fourier transform calculation, where some technical details are deferred to the Appendix. Section 5 treats the case where the life of the option has started and we are possibly for some time period already below the lower barrier or above the upper barrier. The author of [22] has extended the results of the original paper of [8] to incorporate this case. Section 6 discusses the Parisian put contract type, and Sect. 7 summarizes the various contract types that can be derived from the double-sided Parisian knock-in call. One of the contract types that is related to the double-sided Parisian option is the double-sided barrier option, of which pricing by transforms has been done in [12]. More on the relation between double-sided barrier and standard barrier options can be found in [16]. We used the algorithm in [20] to obtain double-sided barrier prices and compare them with double-sided Parisian prices in the section on numerical examples. In Sect. 8 we discuss the Fourier inversion and propose an alternative algorithm. We conclude in Sect. 9 with numerical examples showing various features of the double-sided Parisian option price and Greeks.

\section{The Parisian contract}

Let $(\Omega, \mathcal{F}, \mathbb{P})$ be a probability space with filtration $\left\{\mathcal{F}_{t}\right\}$ and $\left(W_{t}\right)_{t \geq 0}$ a standard Brownian motion with respect to this filtration. By $\left(S_{t}\right)_{t \geq 0}$ we denote the risk-neutral stock price process, given by the classical geometric Brownian motion

$$
S_{t}=S_{0} e^{\left(r-\frac{1}{2} \sigma^{2}\right) t+\sigma W_{t}}
$$

where $r$ and $\sigma$ are the risk-free interest rate and the volatility respectively. In this setup $\mathbb{P}$ is the risk-neutral measure or, equivalently, the pricing measure and not the physical measure. Assuming that there exists a bank account that pays the risk-free interest rate $r$ in a continuously compounded way, the price of an option with a (random) payoff is given by the discounted expectation of that payoff under the pricing measure. The random time $\gamma_{T}^{L}(S)$ measures the last time before $T$ that a process $S$ has been equal to $L$ and is given by

$$
\gamma_{T}^{L}(S):= \begin{cases}\sup \left\{0 \leq t \leq T \mid S_{t}=L\right\} & \text { if } T_{L}(S) \leq T \\ 0 & \text { if } T_{L}(S)>T .\end{cases}
$$

Here $T_{L}(S)$ denotes the hitting time of the level $L$ by the process $S$. Note that $\gamma_{T}^{L}$ is not a stopping time. In case the process is a standard Brownian motion, we suppress the $W$ between brackets that one would expect to appear in (2.1). Now we define the double-sided Parisian stopping time $T_{D_{1}, D_{2}}^{L_{1}-L_{2}+}(S)$ for the levels $L_{1}<L_{2}$ by

$$
T_{D_{1}, D_{2}}^{L_{1}-, L_{2}+}(S):=\min \left(T_{D_{1}}^{L_{1}-}, T_{D_{2}}^{L_{2}+}\right),
$$


where the single-sided Parisian stopping time $T_{D}^{L \pm}$ is given by

$$
T_{D}^{L \pm}:=\inf \left\{t>0 \mid\left(t-\gamma_{t}^{L}(S)\right) 1_{\left\{S_{t} \gtrless L\right\}}>D\right\} .
$$

The stopping time $T_{D_{1}, D_{2}}^{L_{1}-L_{2}+}(S)$ is the first time that the process $S$ stays longer than time $D_{1}$ below level $L_{1}$ or ${ }^{1}$ longer than time $D_{2}$ above level $L_{2}$. The double-sided Parisian knock-in call is a contract that pays off like a standard call, but only in the scenarios where the double-sided Parisian stopping time occurs before the contract expires. The value $V_{\text {DPIC }}$ of this double-sided Parisian knock-in call can be computed as

$$
V_{\text {DPIC }}=e^{-r T} \mathbb{E}\left[\left(S_{T}-K\right)^{+} 1_{\left\{T_{D_{1}, D_{2}}^{L_{1}-, L_{2}+}(S) \leq T\right\}}\right] .
$$

As in [5], we can write this expectation as a sum of two probabilities, namely

$$
V_{\mathrm{DPIC}}=S_{0} P_{r+\sigma^{2}}(T)-K e^{-r T} P_{r}(T),
$$

where

$$
P_{\mu}(T)=\mathbb{P}_{\mu}\left[S_{T}>K ; T_{D_{1}, D_{2}}^{L_{1}-, L_{2}+}(S) \leq T\right], \quad \mu \in \mathbb{R} .
$$

Here $\mu$ denotes the drift of the geometric Brownian motion $S$. The problem of pricing this double-sided Parisian knock-in call is equivalent to computing the probabilities as given in (2.3). The stochastic properties of the stock price process $S$ are entirely determined by the behavior of the underlying Brownian motion, so it is a natural choice to solve the problem in terms of Brownian motion. If we introduce the process $\left(\tilde{W}_{t}\right)_{t \geq 0}$ by

$$
\tilde{W}_{t}:=\frac{r-\frac{1}{2} \sigma^{2}}{\sigma} t+W_{t}=m t+W_{t},
$$

then we can write the stock price $S_{t}$ at $t$ as $S_{0} e^{\sigma \tilde{W}_{t}}$. The events in terms of $S$ can be rewritten into events in terms of $\tilde{W}$ via

$$
\left\{S_{T}>K\right\}=\left\{\tilde{W}_{T}>k\right\}, \quad \text { where } k=\frac{1}{\sigma} \ln \left(\frac{K}{S_{0}}\right) .
$$

In the same manner for $i=1,2$, the levels $L_{i}$ transform into $\ell_{i}$ resulting in $\gamma_{T}^{L_{i}}(S)=\gamma_{T}^{\ell_{i}}(\tilde{W})$. A change of measure allows us to compute the quantity $P_{r}(T)$ by

$$
P_{r}(T)=e^{-\frac{1}{2} m^{2} T} \mathbb{E}\left[e^{m W_{T}} 1_{\left\{W_{T}>k\right\}} 1_{\{\tau \leq T\}}\right],
$$

where we used $\tau$ as a shorthand notation for $T_{D_{1}, D_{2}}^{\ell_{1}-\ell_{2}+}(W)$. The same kind of notation we introduce for $\tau^{+}$and $\tau^{-}$, abbreviating respectively $T_{D_{2}}^{\ell_{2}+}(W)$ and $T_{D_{1}}^{\ell_{1}-}(W)$. By replacing $r$ with $r+\frac{1}{2} \sigma^{2}$ we have to change $m$ in (2.4) to turn (2.5) into a formula for

\footnotetext{
${ }^{1}$ In [17] the double-sided contract type is treated where the process $S$ should stay longer than time $D_{1}$ below level $L_{1}$ and longer than time $D_{2}$ above level $L_{2}$.
} 
$P_{r+\frac{1}{2} \sigma^{2}}(T)$, which we need in the pricing formula (2.2). In the next section we derive formulas for the Laplace transforms of the double-sided Parisian stopping times for standard Brownian motion.

\section{The Laplace transform of the double-sided hitting times}

We start recalling the Brownian meander from the Appendix in [8]. The process $\left(m_{u}^{(t)}\right)_{0 \leq u \leq 1}$ can be defined for every $t>0$ by

$$
m_{u}^{(t)}=\frac{1}{\sqrt{t-\gamma_{t}}}\left|W_{\gamma_{t}+u\left(t-\gamma_{t}\right)}\right|, \quad u \leq 1,
$$

where we suppressed the 0 in the notation of $\gamma_{t}^{0}$. For $t=1$ this process is the Brownian meander. Here we are only interested in $m_{1}^{(t)}$, the final value of the meander which we denote by $n_{t}$ given by

$$
n_{t}=\frac{1}{\sqrt{t-\gamma_{t}}}\left|W_{t}\right|
$$

The $\sigma$-algebra $\mathcal{F}_{\gamma_{t}}^{+}$is generated by the random variable $\operatorname{sgn}\left(W_{t}\right)$ and the variables $\xi_{\gamma_{t}}$, where $\xi$ is a predictable process with respect to the natural Brownian filtration. As pointed out in [8], $n_{t}$ is for every $t>0$ independent of $\mathcal{F}_{\gamma_{t}}^{+}$and therefore independent of the pair $\left(\gamma_{t}, \operatorname{sgn}\left(W_{t}\right)\right)$. Moreover, $n_{t} \stackrel{d}{=} N$, where $N$ has the density

$$
\mathbb{P}[N \in \mathrm{d} x]=x e^{-\frac{x^{2}}{2}} 1_{\{x \geq 0\}} \mathrm{d} x,
$$

and for later on it is useful to define the function $\Psi_{c}$ for $c \geq 0$ by

$$
\Psi_{c}(z):=\mathbb{E}\left[e^{z N} 1_{\{N \geq c\}}\right]=e^{-\frac{c^{2}}{2}+z c}+z \sqrt{2 \pi} e^{\frac{z^{2}}{2}} \mathcal{N}(z-c),
$$

where $\mathcal{N}$ is the CDF of the standard normal distribution. We abbreviate $\Psi_{0}$ by $\Psi$ and $\Psi-\Psi_{c}$ by $\tilde{\Psi}_{c}$. Now consider the meander at time $t$ away from level $\ell$ and denote its final value by $n_{t}^{\ell}$ given by

$$
n_{t}^{\ell}=\frac{1_{\left\{T_{\ell}<t\right\}}}{\sqrt{t-\gamma_{t}^{\ell}}}\left|W_{t}-\ell\right|,
$$

where $T_{\ell}$ denotes the first hitting time of the level $\ell$ by Brownian motion. It follows from the strong Markov property and the independence of $n_{t}$ and the pair $\left(\operatorname{sgn}\left(W_{t}\right), \gamma_{t}\right)$ that for all bounded, measurable functions $f$ and $g$, we have

$$
\begin{aligned}
\mathbb{E} & {\left[f\left(n_{t}^{\ell}\right) g\left(\operatorname{sgn}\left(W_{t}-\ell\right), \gamma_{t}^{\ell}\right) 1_{\left\{T_{\ell}<t\right\}} \mid \mathcal{F}_{T_{\ell}}\right] } \\
& =1_{\left\{T_{\ell}<t\right\}} \mathbb{E}^{\ell}\left[f\left(\frac{\left|W_{t-s}-\ell\right|}{\sqrt{t-\left(\gamma_{t-s}^{\ell}+s\right)}}\right) g\left(\operatorname{sgn}\left(W_{t-s}-\ell\right), \gamma_{t-s}^{\ell}+s\right)\right]_{s=T_{\ell}}
\end{aligned}
$$




$$
\begin{aligned}
& =1_{\left\{T_{\ell}<t\right\}} \mathbb{E}\left[f\left(\frac{\left|W_{t-s}\right|}{\sqrt{t-s-\gamma_{t-s}}}\right) g\left(\operatorname{sgn}\left(W_{t-s}\right), \gamma_{t-s}+s\right)\right]_{s=T_{\ell}} \\
& =1_{\left\{T_{\ell}<t\right\}} \mathbb{E}[f(N)] \mathbb{E}\left[g\left(\operatorname{sgn}\left(W_{t-s}\right), \gamma_{t-s}+s\right)\right]_{s=T_{\ell}} \\
& =\mathbb{E}[f(N)] \mathbb{E}\left[g\left(\operatorname{sgn}\left(W_{t}-\ell\right), \gamma_{t}^{\ell}\right) 1_{\left\{T_{\ell}<t\right\}} \mid \mathcal{F}_{T_{\ell}}\right] .
\end{aligned}
$$

Here $\mathbb{E}^{\ell}$ denotes the expectation under the measure under which Brownian motion $W$ starts from level $\ell$. Hence, conditionally on hitting the level $\ell$ before time $t, n_{t}^{\ell}$ has the same distribution as $N$ and $n_{t}^{\ell}$ is independent of the pair $\left(\operatorname{sgn}\left(W_{t}-\ell\right), \gamma_{t}^{\ell}\right)$. In Appendix A.6 we show that (3.3) also holds for $\tau^{+}$. Now we can construct another process $\mu^{\ell}$ by

$$
\mu_{t}^{\ell}=1_{\left\{T_{\ell}<t\right\}} \operatorname{sgn}\left(W_{t}-\ell\right) \sqrt{t-\gamma_{t}^{\ell}},
$$

which is, given $T_{\ell}<t$, conditionally independent of $n_{t}^{\ell}$, so we can use it to decompose the Brownian motion into two independent parts, namely

$$
1_{\left\{T_{\ell<t\}}\right.}\left(W_{t}-\ell\right)=n_{t}^{\ell} \mu_{t}^{\ell}
$$

Now we construct the $\sigma$-algebra $\mathcal{H}_{\tau}$ that contains the information of the processes $\mu^{\ell_{i}}$ and $\gamma^{\ell_{i}}$ for $i=1,2$ at the random time $\tau$ by

$$
\mathcal{H}_{\tau}=\sigma\left(\mu_{\tau}^{\ell_{1}}, \mu_{\tau}^{\ell_{2}}, \gamma_{\tau}^{\ell_{1}}, \gamma_{\tau}^{\ell_{2}}\right)
$$

where we assume $\ell_{1} \leq 0 \leq \ell_{2}$. Note that the events $\left\{\tau^{+}<\tau^{-}\right\}$and $\left\{\tau^{-}<\tau^{+}\right\}$can be constructed from these random variables via

$$
\left\{\tau^{-}<\tau^{+}\right\}=\left\{\mu_{\tau}^{\ell_{1}}<0\right\}, \quad\left\{\tau^{+}<\tau^{-}\right\}=\left\{\mu_{\tau}^{\ell_{2}}>0\right\},
$$

and therefore they are $\mathcal{H}_{\tau}$-measurable. The equation

$$
\tau=\left(\gamma_{\tau}^{\ell_{1}}+D_{1}\right) 1_{\left\{\tau^{-}<\tau^{+}\right\}}+\left(\gamma_{\tau}^{\ell_{2}}+D_{2}\right) 1_{\left\{\tau^{+}<\tau^{-}\right\}}
$$

shows that $\tau$ is also $\mathcal{H}_{\tau}$-measurable. The following lemma states conditional independence between $n_{\tau}^{\ell_{2}}$ and $\mathcal{H}_{\tau}$.

Lemma 3.1 The following equation holds for any bounded measurable function $f$ :

$$
\mathbb{E}\left[1_{\left\{\tau^{+}<\tau^{-}\right\}} f\left(n_{\tau}^{\ell_{2}}\right) \mid \mathcal{H}_{\tau}\right]=1_{\left\{\tau^{+}<\tau^{-}\right\}} \mathbb{E}[f(N)] \text { a.s. }
$$

Proof We have to show that for any $H \in \mathcal{H}_{\tau}$, we have

$$
\int_{H} 1_{\left\{\tau^{+}<\tau^{-}\right\}} f\left(n_{\tau}^{\ell_{2}}\right) \mathrm{d} \mathbb{P}=\mathbb{E}[f(N)] \mathbb{P}\left[H ; \tau^{+}<\tau^{-}\right],
$$

and directly from the construction of $\mathcal{H}_{\tau}$, this is equivalent to showing that for any bounded measurable function $g$, we have 


$$
\begin{aligned}
\mathbb{E} & {\left[1_{\left\{\tau^{+}<\tau^{-}\right\}} f\left(n_{\tau}^{\ell_{2}}\right) g\left(\gamma_{\tau}^{\ell_{1}}, \gamma_{\tau}^{\ell_{2}}, \mu_{\tau}^{\ell_{1}}, \mu_{\tau}^{\ell_{2}}\right)\right] } \\
& =\mathbb{E}[f(N)] \mathbb{E}\left[1_{\left\{\tau^{+}<\tau^{-}\right\}} g\left(\gamma_{\tau}^{\ell_{1}}, \gamma_{\tau}^{\ell_{2}}, \mu_{\tau}^{\ell_{1}}, \mu_{\tau}^{\ell_{2}}\right)\right] .
\end{aligned}
$$

On the set $\left\{\tau^{+}<\tau^{-}\right\}$we have $\mu_{\tau}^{\ell_{2}}=\sqrt{D_{2}}$ and $\mu_{\tau}^{\ell_{1}}$ can be expressed in terms of $\gamma_{\tau}^{\ell_{1,2}}$ via

$$
\mu_{\tau}^{\ell_{1}}=1_{\left\{\gamma_{\tau}^{\ell_{1}}>0\right\}} \sqrt{D_{2}+\left(\gamma_{\tau}^{\ell_{2}}-\gamma_{\tau}^{\ell_{1}}\right)} .
$$

So we can rewrite the function $g$ as a function $\tilde{g}$ of $\gamma_{\tau}^{\ell_{1,2}}$ only. After replacing $\left\{\tau^{+}<\tau^{-}\right\}$by $\left\{\mu_{\tau}^{\ell_{2}}>0\right\}$ as in (3.4), it remains to show that

$$
\mathbb{E}\left[1_{\left\{\mu_{\tau}^{\ell_{2}}>0\right\}} f\left(n_{\tau}^{\ell_{2}}\right) \tilde{g}\left(\gamma_{\tau}^{\ell_{1}}, \gamma_{\tau}^{\ell_{2}}\right)\right]=\mathbb{E}[f(N)] \mathbb{E}\left[1_{\left\{\mu_{\tau}^{\ell_{2}}>0\right\}} \tilde{g}\left(\gamma_{\tau}^{\ell_{1}}, \gamma_{\tau}^{\ell_{2}}\right)\right] .
$$

Now by (3.3) both $\mu_{\tau}^{\ell_{2}}$ and $\gamma_{\tau}^{\ell_{2}}$ are conditionally independent of $n_{\tau}^{\ell_{2}}$, so (3.5) would hold if $\tilde{g}$ were a function of $\gamma_{\tau}^{\ell_{2}}$ only. This is true for the case $\ell_{1}=0=\ell_{2}$ for which $\gamma_{\tau}^{\ell_{1}}=\gamma_{\tau}^{\ell_{2}}$. Therefore it remains to prove for the cases $\ell_{1} \leq 0<\ell_{2}$ and $\ell_{1}<0 \leq \ell_{2}$ that on the set $\left\{\mu_{\tau}^{\ell_{2}}>0\right\}$ also $\gamma_{\tau}^{\ell_{1}}$ is independent of $n_{\tau}^{\ell_{2}}$. Denote by $\ell_{c}$ the midpoint of the barriers, i.e.,

$$
\ell_{c}=\frac{\ell_{1}+\ell_{2}}{2}
$$

and define the sequence of stopping times $T^{(n)}, n=0,1, \ldots$, by $T^{(0)}=0$ and

$$
T^{(n+1)}=\inf \left\{t>T^{(n)} \mid W_{t}=\ell_{c} \text { and } W_{s} \in\left\{\ell_{1}, \ell_{2}\right\} \text { for some } T^{(n)} \leq s \leq t\right\} .
$$

In the sequel of the proof, we shall use these stopping times to restart Brownian motion by a strong Markov argument at exactly this midpoint. Note that $\tau^{+}$is always between two of the stopping times, $T^{(n)}$ and $T^{(n+1)}$. Moreover, $\tau^{-}$cannot be between the same two stopping times as $\tau^{+}$, so we can write for any bounded measurable functions $f$ and $h$ that

$$
\begin{aligned}
\mathbb{E} & {\left[1_{\left\{\mu_{\tau}^{\ell_{2}}>0\right\}} f\left(n_{\tau}^{\ell_{2}}\right) h\left(\gamma_{\tau}^{\ell_{1}}\right)\right] } \\
& =\sum_{n=0}^{\infty} \mathbb{E}\left[1_{\left\{T^{(n)}<\tau\right\}} h\left(\gamma_{T^{(n)}}^{\ell_{1}}\right) \mathbb{E}\left[1_{\left\{\tau<T^{(n+1)}\right\}} 1_{\left\{\mu_{\tau}^{\ell_{2}}>0\right\}} f\left(n_{\tau}^{\ell_{2}}\right) \mid \mathcal{F}_{T^{(n)}}\right]\right],
\end{aligned}
$$

where we used that on the set $\left\{\tau^{+}<\tau^{-}\right\} \cap\left\{T^{(n)}<\tau<T^{(n+1)}\right\}$ we have $\gamma_{\tau}^{\ell_{1}}<T^{(n)}$ and therefore $\gamma_{\tau}^{\ell_{1}}=\gamma_{T^{(n)}}^{\ell_{1}}$. Now we can use the strong Markov property to restart Brownian motion in $\ell_{c}$ at time $T^{(n)}$ for $n>0$ and obtain

$$
\begin{aligned}
\sum_{n=0}^{\infty} & \mathbb{E} \\
= & \left.1_{\left\{T^{(n)}<\tau\right\}} h\left(\gamma_{T^{(n)}}^{\ell_{1}}\right) \mathbb{E}\left[1_{\left\{\tau<T^{(n+1)}\right\}} 1_{\left\{\mu_{\tau}^{\left.\ell_{2}>0\right\}}\right.} f\left(n_{\tau}^{\ell_{2}}\right) \mid \mathcal{F}_{T^{(n)}}\right]\right] \\
= & \mathbb{E}\left[1_{\left\{T^{(0)}<\tau\right\}} h\left(\gamma_{T^{(0)}}^{\ell_{1}}\right) \mathbb{E}\left[1_{\left\{\tau<T^{(1)}\right\}} 1_{\left\{\mu_{\tau}^{\left.\ell_{2}>0\right\}}\right.} f\left(n_{\tau}^{\ell_{2}}\right)\right]\right] \\
& +\sum_{n=1}^{\infty} \mathbb{E}\left[1_{\left\{T^{(n)}<\tau\right\}} h\left(\gamma_{T^{(n)}}^{\ell_{1}}\right) \mathbb{E}^{\ell_{c}}\left[1_{\left\{\tau<T^{(1)}\right\}} 1_{\left\{\mu_{\tau}^{\ell_{2}}>0\right\}} f\left(n_{\tau}^{\ell_{2}}\right)\right]\right]
\end{aligned}
$$




$$
\begin{aligned}
= & \mathbb{E}\left[1_{\left\{T^{(0)}<\tau\right\}} h\left(\gamma_{T^{(0)}}^{\ell_{1}}\right) \mathbb{E}\left[1_{\left\{\tau^{+}<T^{(1)}\right\}} f\left(n_{\tau^{+}}^{\ell_{2}}\right)\right]\right] \\
& +\sum_{n=1}^{\infty} \mathbb{E}\left[1_{\left\{T^{(n)}<\tau\right\}} h\left(\gamma_{T^{(n)}}^{\ell_{1}}\right) \mathbb{E}^{\ell_{c}}\left[1_{\left\{\tau^{+}<T^{(1)}\right\}} f\left(n_{\tau^{+}}^{\ell_{2}}\right)\right]\right],
\end{aligned}
$$

where we used in the last equality that the event $\left\{\tau^{+}<T^{(1)}\right\}$ equals the intersection of the events $\left\{\tau<T^{(1)}\right\}$ and $\left\{\tau^{+}<\tau^{-}\right\}$. Note that $T^{(0)}$ is treated separately from $T^{(n)}$ for $n>0$. The equation still holds in the subtle case $\ell_{1}=0$, because in that case $\mathbb{P}\left[\tau_{+}<T^{(1)}\right]=0$. By (3.3) we can establish the independence of $\tau^{+}$and $n_{\tau^{+}}^{\ell_{2}}$, and therefore the expectation of $n_{\tau^{+}}^{\ell_{2}}$ can be taken out of the sum to give

$$
\begin{aligned}
\mathbb{E}\left[1_{\left\{T^{(0)}<\tau\right\}} h\left(\gamma_{T^{(0)}}^{\ell_{1}}\right) \mathbb{E}\left[1_{\left\{\tau^{+}<T^{(1)}\right\}} f\left(n_{\tau^{+}}^{\ell_{2}}\right)\right]\right] \\
+\sum_{n=1}^{\infty} \mathbb{E}\left[1_{\left\{T^{(n)}<\tau\right\}} h\left(\gamma_{T^{(n)}}^{\ell_{1}}\right) \mathbb{E}^{\ell_{c}}\left[1_{\left\{\tau^{+}<T^{(1)}\right\}} f\left(n_{\tau^{+}}^{\ell_{2}}\right)\right]\right] \\
=\mathbb{E}[f(N)]\left\{\mathbb{E}\left[1_{\left\{T^{(0)}<\tau\right\}} h\left(\gamma_{T^{(0)}}^{\ell_{1}}\right) \mathbb{E}\left[1_{\left\{\tau^{+}<T^{(1)}\right\}}\right]\right]\right. \\
\left.+\sum_{n=1}^{\infty} \mathbb{E}\left[1_{\left\{T^{(n)}<\tau\right\}} h\left(\gamma_{T^{(n)}}^{\ell_{1}}\right) \mathbb{E}^{\ell_{c}}\left[1_{\left\{\tau^{+}<T^{(1)}\right\}}\right]\right\}\right\} \\
=\mathbb{E}[f(N)]\left\{\mathbb{E}\left[1_{\left\{T^{(0)}<\tau\right\}} h\left(\gamma_{T^{(0)}}^{\ell_{1}}\right) \mathbb{E}\left[1_{\left\{\tau^{+}<T^{(1)}\right\}} \mid \mathcal{F}_{T^{(0)}}\right]\right]\right. \\
\left.+\sum_{n=1}^{\infty} \mathbb{E}\left[1_{\left\{T^{(n)}<\tau\right\}} h\left(\gamma_{T^{(n)}}^{\ell_{1}}\right) \mathbb{E}\left[1_{\left\{\tau^{+}<T^{(n+1)}\right\}} \mid \mathcal{F}_{T^{(n)}}\right]\right]\right\} \\
=\mathbb{E}[f(N)] \sum_{n=0}^{\infty} \mathbb{E}\left[1_{\left\{T^{(n)}<\tau\right\}} h\left(\gamma_{T^{(n)}}^{\ell_{1}}\right) 1_{\left\{\tau^{+}<T^{(n+1)}\right\}}\right] .
\end{aligned}
$$

In the final step we use that

$$
\left\{T^{(n)}<\tau\right\} \cap\left\{\tau^{+}<T^{(n+1)}\right\}=\left\{T^{(n)}<\tau<T^{(n+1)}\right\} \cap\left\{\tau^{+}<\tau^{-}\right\} .
$$

Moreover, on this set the level $\ell_{1}$ is not touched within the time interval $\left(T^{(n)}, T^{(n+1)}\right)$. Therefore we can replace $\gamma_{T^{(n)}}^{\ell_{1}}$ by $\gamma_{\tau}^{\ell_{1}}$, altogether resulting in

$$
\begin{aligned}
\mathbb{E} & {[f(N)] \sum_{n=0}^{\infty} \mathbb{E}\left[1_{\left\{T^{(n)}<\tau\right\}} h\left(\gamma_{T^{(n)}}^{\ell_{1}}\right) 1_{\left\{\tau^{+}<T^{(n+1)}\right\}}\right] } \\
= & \mathbb{E}[f(N)] \mathbb{E}\left[h\left(\gamma_{\tau}^{\ell_{1}}\right) 1_{\left\{\mu_{\tau}^{\ell_{2}}>0\right\}}\right],
\end{aligned}
$$

which yields the desired independence result. 
We are interested in the Laplace transform of $\tau$ and we have the following theorem on $\mathbb{E}_{+}(\lambda)$ and $\mathbb{E}_{-}(\lambda)$, the restricted Laplace transforms of $\tau$ defined by

$$
\mathbb{E}_{+}(\lambda):=\mathbb{E}\left[e^{-\frac{1}{2} \lambda^{2} \tau} 1_{\left\{\tau^{+}<\tau^{-}\right\}}\right] \quad \text { and } \quad \mathbb{E}_{-}(\lambda):=\mathbb{E}\left[e^{-\frac{1}{2} \lambda^{2} \tau} 1_{\left\{\tau^{-}<\tau^{+}\right\}}\right]
$$

Theorem 3.2 For the restricted Laplace transforms $\mathbb{E}_{+}(\lambda)$ and $\mathbb{E}_{-}(\lambda)$ of $\tau$, we have

$$
\begin{aligned}
& \mathbb{E}_{+}(\lambda)=\frac{e^{\lambda \ell_{1}} \Psi\left(-\lambda_{1}\right)-e^{-\lambda \ell_{1}} \Psi\left(\lambda_{1}\right)}{e^{\lambda\left(\ell_{1}-\ell_{2}\right)} \Psi\left(-\lambda_{1}\right) \Psi\left(-\lambda_{2}\right)-e^{\lambda\left(\ell_{2}-\ell_{1}\right)} \Psi\left(\lambda_{1}\right) \Psi\left(\lambda_{2}\right)}, \\
& \mathbb{E}_{-}(\lambda)=\frac{e^{-\lambda \ell_{2}} \Psi\left(-\lambda_{2}\right)-e^{\lambda \ell_{2}} \Psi\left(\lambda_{2}\right)}{e^{\lambda\left(\ell_{1}-\ell_{2}\right)} \Psi\left(-\lambda_{1}\right) \Psi\left(-\lambda_{2}\right)-e^{\lambda\left(\ell_{2}-\ell_{1}\right)} \Psi\left(\lambda_{1}\right) \Psi\left(\lambda_{2}\right)},
\end{aligned}
$$

where we used $\lambda_{i}=\lambda \sqrt{D_{i}}$ for $i=1,2$ to shorten the notation.

Proof The first step of the proof consists of an application of the optional sampling theorem to the martingale $M$ defined by $M_{t}=e^{-\frac{1}{2} \lambda^{2} t+\lambda W_{t}}$ to give

$$
\begin{aligned}
1 & =\mathbb{E}\left[e^{-\frac{1}{2} \lambda^{2} \tau+\lambda W_{\tau}}\right] \\
& =\mathbb{E}\left[e^{-\frac{1}{2} \lambda^{2} \tau+\lambda W_{\tau}} 1_{\left\{\tau^{+}<\tau^{-}\right\}}\right]+\mathbb{E}\left[e^{-\frac{1}{2} \lambda^{2} \tau+\lambda W_{\tau}} 1_{\left\{\tau^{-}<\tau^{+}\right\}}\right],
\end{aligned}
$$

where the optional sampling theorem holds because Appendix A.3 shows that $M^{\tau}$, the $\tau$-stopped version of the martingale $M$, is a uniformly integrable martingale. The expectation in (3.7) on the left-hand side can be computed as

$$
\begin{aligned}
\mathbb{E} & {\left[e^{-\frac{1}{2} \lambda^{2} \tau+\lambda W_{\tau}} 1_{\left\{\tau^{+}<\tau^{-}\right\}}\right] } \\
& =\mathbb{E}\left[e^{-\frac{1}{2} \lambda^{2} \tau+\lambda\left(\mu_{\tau}^{\ell_{2}} n_{\tau^{\ell}}^{\ell_{2}}+\ell_{2}\right)} 1_{\left\{\tau^{+}<\tau^{-}\right\}}\right] \\
& =e^{\lambda \ell_{2}} \mathbb{E}\left[e^{-\frac{1}{2} \lambda^{2} \tau} 1_{\left\{\tau^{+}<\tau^{-}\right\}} \mathbb{E}\left[e^{\lambda \sqrt{D_{2}} n_{\tau}} \mid \mathcal{H}_{\tau}\right]\right] \\
& =e^{\lambda \ell_{2}} \mathbb{E}\left[e^{\lambda \sqrt{D_{2}} N}\right] \mathbb{E}\left[e^{-\frac{1}{2} \lambda^{2} \tau} 1_{\left\{\tau^{+}<\tau^{-}\right\}}\right]=e^{\lambda \ell_{2}} \Psi\left(\lambda \sqrt{D_{2}}\right) \mathbb{E}_{+}(\lambda),
\end{aligned}
$$

where Lemma 3.1 is used to obtain the third line from the second. By the symmetry of Brownian motion we find for the right-hand side expectation in (3.7) the expression

$$
\mathbb{E}\left[e^{-\frac{1}{2} \lambda^{2} \tau+\lambda W_{\tau}} 1_{\left\{\tau^{+}>\tau^{-}\right\}}\right]=e^{\lambda \ell_{1}} \Psi\left(-\lambda \sqrt{D_{1}}\right) \mathbb{E}_{-}(\lambda)
$$

Note that $\mathbb{E}_{+}(\lambda)$ and $\mathbb{E}_{-}(\lambda)$ are even functions of $\lambda$ and the theorem is proved by solving the set of equations obtained by plugging (3.8) and (3.9) into (3.7) for $\pm \lambda$.

By taking the limit for $\lambda \rightarrow 0$ in (3.6), we can derive the following corollary from the theorem. 
Corollary 3.3 The probability that Brownian motion will spend time $D_{1}$ below level $\ell_{1}$ before it spends $D_{2}$ above level $\ell_{2}$ is given by the formula

$$
\mathbb{P}\left[\tau^{-}<\tau^{+}\right]=\frac{\ell_{2} \sqrt{\frac{2}{\pi}}+\sqrt{D_{2}}}{\left(\ell_{2}-\ell_{1}\right) \sqrt{\frac{2}{\pi}}+\sqrt{D_{1}}+\sqrt{D_{2}}}, \quad \ell_{1}<0<\ell_{2} .
$$

Remark 3.4 By taking the limits $\ell_{2} \downarrow 0$ and $\ell_{1} \uparrow 0$ in (3.10), we get for Brownian motion that the probability that a positive excursion of length $D_{2}$ happens before a negative excursion of length $D_{1}$ occurs is equal to

$$
\frac{\sqrt{D_{1}}}{\sqrt{D_{1}}+\sqrt{D_{2}}}
$$

which is a remarkably simple expression. In the same way as in [21] one could use excursion theory as another way to obtain this probability. In [10] the authors arrive at similar equations.

Now we have computed the Laplace transforms of the Parisian stopping times for a standard Brownian motion, we return in the next section to the original problem of deriving Fourier transforms for the probabilities related to the double-sided Parisian knock-in call option contract.

\section{Calculating the Fourier transform}

We recall that the relevant quantity to compute in order to price Parisian options maturing at time $T$ is the probability $P_{r}(T)$ given by (2.3). Also note that the formulas for $P_{r+\frac{1}{2} \sigma^{2}}(T)$ appear to be similar, except that a different value for $m$ is needed. Here we calculate its Fourier transform $\phi$ in the parameter $T$ and actual numbers can be obtained by numerical inversion. In this section we assume $L_{1} \leq S_{0} \leq L_{2}$ or, stated in terms of standard Brownian motion, $\ell_{1} \leq 0 \leq \ell_{2}$. The case where the initial stock price $S_{0}$ is above $L_{2}$ or below $L_{1}$ is treated in Sect. 5. As a function of time, the probability $P_{r}(T)$ is non-decreasing, and in order to assure integrability we introduce an exponential damping factor $a>0$. We have for $\phi$ that

$$
\phi(v)=\int_{0}^{\infty} e^{i v T} e^{-a T} P_{r}(T) \mathrm{d} T .
$$

Now we can substitute (2.5) for $P_{r}$, use $\alpha=a+\frac{1}{2} m^{2}$, and split the event $\{\tau \leq T\}$ into the parts where the Parisian constraint is fulfilled above the upper level or below the lower level to obtain

$$
\begin{aligned}
\phi(v) & =\int e^{(i v-\alpha) T} \mathbb{E}\left[e^{m W_{T}} 1_{\left\{W_{T}>k\right\}} 1_{\{\tau \leq T\}}\left(1_{\left\{\tau^{+}<\tau^{-}\right\}}+1_{\left\{\tau^{-}<\tau^{+}\right\}}\right)\right] \mathrm{d} T \\
& =: \phi_{+}(v)+\phi_{-}(v) .
\end{aligned}
$$


Of course, $\phi$ depends on the value of the strike, the barriers and the times that have to be spent below or above these barriers, so a more precise notation would be $\phi\left(v ; k, \ell_{1}, D_{1}, \ell_{2}, D_{2}\right)$. Here we try to keep the notation as simple as possible without being ambiguous. The following lemma links the results from the previous section to $\phi_{+}$and $\phi_{-}$.

Lemma 4.1 The Fourier transforms $\phi_{+}$and $\phi_{-}$can be written as

$$
\begin{aligned}
& \phi_{+}(v)=\mathbb{E}_{+}\left(\tilde{v}_{\alpha}\right) \mathbb{E}\left[\int_{0}^{\infty} e^{(i v-\alpha) \rho} h\left(\rho, \ell_{2}+\sqrt{D_{2}} N\right) \mathrm{d} \rho\right], \\
& \phi_{-}(v)=\mathbb{E}_{-}\left(\tilde{v}_{\alpha}\right) \mathbb{E}\left[\int_{0}^{\infty} e^{(i v-\alpha) \rho} h\left(\rho, \ell_{1}-\sqrt{D_{1}} N\right) \mathrm{d} \rho\right],
\end{aligned}
$$

where $N$ is the random variable with density (3.1) and

$$
h(\rho, w)=\mathbb{E}\left[e^{m\left(W_{\rho}+w\right)} 1_{\left\{W_{\rho}+w>k\right\}}\right] \quad \text { and } \quad \tilde{v}_{\alpha}=\sqrt{2(\alpha-i v)} .
$$

Proof We give the proof for $\phi_{+}$which, after an application of Fubini, the substitution $\rho=T-\tau$ and the strong Markov property of Brownian motion, can be written as

$$
\phi_{+}(v)=\mathbb{E}\left[1_{\left\{\tau^{+}<\tau^{-}\right\}} \int_{0}^{\infty} e^{(i v-\alpha)(\rho+\tau)} \mathbb{E}\left[e^{m\left(W_{\rho}+w\right)} 1_{\left\{W_{\rho}+w>k\right\}}\right]_{w=W_{\tau}} \mathrm{d} \rho\right]
$$

Given $\tau^{+}<\tau^{-}$, by Lemma 3.1, the stopping time $\tau$ and the value $W_{\tau}$ of Brownian motion are independent and, moreover, $W_{\tau} \stackrel{d}{=} \ell_{2}+\sqrt{D_{2}} N$, which proves the result for $\phi_{+}$. By the symmetry of Brownian motion, the proof for $\phi_{-}$proceeds along the same lines, where we remark that now $W_{\tau} \stackrel{d}{=} \ell_{1}-\sqrt{D_{1}} N$, given $\tau^{-}<\tau^{+}$.

The quantities $\mathbb{E}_{+}$and $\mathbb{E}_{-}$in (4.3) and (4.4) are given by Theorem 3.2, so we proceed to calculate the remaining expectations. Let $c_{1}$ and $c_{2}$ be real numbers and denote by $E\left(c_{1}, c_{2}\right)$ the relevant expectation for Lemma 4.1:

$$
E\left(c_{1}, c_{2}\right)=\mathbb{E}\left[\int_{0}^{\infty} e^{(i v-\alpha) \rho} h\left(\rho, c_{1}+c_{2} N\right) \mathrm{d} \rho\right]
$$

The following lemma computes this expectation for special case of $c_{1}$ and $c_{2}$.

Lemma 4.2 The following holds for the expectation $E\left(c_{1}, c_{2}\right)$ :

$$
E\left(c_{1}, c_{2}\right)= \begin{cases}\frac{e^{\left(m-\tilde{v}_{\alpha}\right) k+\tilde{v}_{\alpha} c_{1}}}{\tilde{v}_{\alpha}\left(\tilde{v}_{\alpha}-m\right)} \mathbb{E}\left[e^{\tilde{v}_{\alpha} c_{2} N}\right], & k>c_{1}, c_{2}<0, \\ \frac{2 e^{m c_{1}}}{\tilde{v}_{\alpha}^{2}-m^{2}} \mathbb{E}\left[e^{m c_{2} N}\right]-\frac{e^{k\left(m+\tilde{v}_{\alpha}\right)-c_{1} \tilde{v}_{\alpha}}}{\tilde{v}_{\alpha}\left(m+\tilde{v}_{\alpha}\right)} \mathbb{E}\left[e^{-\tilde{v}_{\alpha} c_{2} N}\right], & k<c_{1}, c_{2}>0 .\end{cases}
$$


Proof The expectation $E\left(c_{1}, c_{2}\right)$ can be written out as

$$
\begin{aligned}
E\left(c_{1}, c_{2}\right)= & \int_{0}^{\infty} u e^{-\frac{u^{2}}{2}} \int_{k-c_{1}-c_{2} u}^{\infty} e^{m\left(x+c_{2} u+c_{1}\right)} \\
& \times \int_{0}^{\infty} e^{(i v-\alpha) \rho} \frac{1}{\sqrt{2 \pi \rho}} e^{-\frac{x^{2}}{2 \rho}} \mathrm{d} \rho \mathrm{d} x \mathrm{~d} u,
\end{aligned}
$$

where we integrate over the densities of $W_{\rho}$ and $N$ and change the order of integration with Fubini. Calculation of the inner $\rho$-integral yields

$$
\begin{aligned}
\int_{0}^{\infty} e^{(i v-\alpha) \rho} \frac{1}{\sqrt{2 \pi \rho}} e^{-\frac{x^{2}}{2 \rho}} \mathrm{d} \rho & =\int_{0}^{\infty} e^{(i v-\alpha) \rho} \int_{|x|}^{\infty} \frac{y}{\sqrt{2 \pi \rho^{3}}} e^{-\frac{y^{2}}{2 \rho}} \mathrm{d} y \mathrm{~d} \rho \\
& =\int_{|x|}^{\infty} e^{-y \sqrt{2(\alpha-i v)}} \mathrm{d} y=\frac{e^{-|x| \tilde{v}_{\alpha}}}{\tilde{v}_{\alpha}} .
\end{aligned}
$$

Here we used the complex continuation of the Laplace transform of the first hitting time of $y$ by standard Brownian motion; see Chap. 2 of [15] for example. Now we combine the previous two equations to arrive at

$$
E\left(c_{1}, c_{2}\right)=\frac{e^{m c_{1}}}{\tilde{v}_{\alpha}} \int_{0}^{\infty} u e^{-\frac{u^{2}}{2}+c_{2} m u} \int_{k-c_{1}-c_{2} u}^{\infty} e^{m x-\tilde{v}_{\alpha}|x|} \mathrm{d} x \mathrm{~d} u .
$$

The inner integral of (4.7) converges as a result of the definition of $\alpha$ just below Equation (4.1) and must be computed for each of the cases $k>c_{1}, c_{2}<0$ and $k<c_{1}, c_{2}>0$ separately. For the case $k>c_{1}, c_{2}<0$ we have that $k-c_{1}-c_{2} u \geq 0$ for every $u \geq 0$, so we simply can replace $|x|$ by $x$ in (4.7) and compute the integral. The resulting integral contains the density of $N$ as given in (3.1) and therefore equals the expectation as stated in the lemma. The case $k<c_{1}, c_{2}>0$ is slightly more complicated, as $k-c_{1}-c_{2} u \leq 0$ for every $u \geq 0$. Now the integral in (4.7) containing $|x|$ needs to be split up into an integral over $x \in\left[k-c_{1}-c_{2} u, 0\right]$ and an integral over $x \in(0, \infty)$ replacing $|x|$ by $-x$ and $x$ respectively.

We have now all the ingredients we need to calculate $\phi_{+}$and $\phi_{-}$in the following special cases.

Corollary 4.3 The following expressions hold for $\phi_{+}$and $\phi_{-}$in case $\ell_{1} \leq 0 \leq \ell_{2}$ and $\ell_{1} \leq k \leq \ell_{2}$ :

$$
\begin{aligned}
& \phi_{+}(v)=\mathbb{E}_{+}\left(\tilde{v}_{\alpha}\right)\left(\frac{2 e^{m \ell_{2}} \Psi\left(m \sqrt{D_{2}}\right)}{\tilde{v}_{\alpha}^{2}-m^{2}}-\frac{e^{k\left(m+\tilde{v}_{\alpha}\right)-\ell_{2} \tilde{v}_{\alpha} \Psi\left(-\tilde{v}_{\alpha} \sqrt{D_{2}}\right)}}{\tilde{v}_{\alpha}\left(m+\tilde{v}_{\alpha}\right)}\right), \\
& \ell_{2} \geq k, \\
& \phi_{-}(v)=\mathbb{E}_{-}\left(\tilde{v}_{\alpha}\right) \frac{e^{\left(m-\tilde{v}_{\alpha}\right) k+\tilde{v}_{\alpha} \ell_{1} \Psi\left(-\tilde{v}_{\alpha} \sqrt{D_{1}}\right)}}{\tilde{v}_{\alpha}\left(\tilde{v}_{\alpha}-m\right)}, \quad \ell_{1} \leq k .
\end{aligned}
$$


Proof The corollary directly follows by plugging the result of Lemma 4.2 into Lemma 4.1 and using the special function $\Psi$ defined in (3.2) for the expectation in Lemma 4.2.

Adding $\phi_{-}$and $\phi_{+}$gives an expression for $\phi$. We remark that the expressions are only valid for $L_{1} \leq K, S_{0} \leq L_{2}$. In order to get expressions for the general case, we need a formula for $\phi_{+}$in case $k>\ell_{2}$ and a formula for $\phi_{-}$in case $k<\ell_{1}$. In order to get there, we need to extend Lemma 4.2 to the cases $k<c_{1}, c_{2}<0$ and $k>c_{1}, c_{2}>0$, which is rather involved and therefore deferred to the Appendix. Here we just state the results as they follow from Lemma A.1 in Appendix A.1: For $\phi_{+}$, we get

$$
\begin{aligned}
\phi_{+}(v)= & \mathbb{E}_{+}\left(\tilde{v}_{\alpha}\right)\left(\frac{e^{\left(m-\tilde{v}_{\alpha}\right) k+\ell_{2} \tilde{v}_{\alpha}} \tilde{\Psi}_{u_{2}^{*}}\left(\tilde{v}_{\alpha} \sqrt{D_{2}}\right)}{\tilde{v}_{\alpha}\left(\tilde{v}_{\alpha}-m\right)}+\frac{2 e^{m \ell_{2}} \Psi_{u_{2}^{*}}\left(m \sqrt{D_{2}}\right)}{\tilde{v}_{\alpha}^{2}-m^{2}}\right. \\
& \left.-\frac{e^{\left(\tilde{v}_{\alpha}+m\right) k-\ell_{2} \tilde{v}_{\alpha}} \Psi_{u_{2}^{*}}\left(-\tilde{v}_{\alpha} \sqrt{D_{2}}\right)}{\tilde{v}_{\alpha}\left(\tilde{v}_{\alpha}+m\right)}\right), \quad k>\ell_{2},
\end{aligned}
$$

and for $\phi_{-}$, we obtain

$$
\begin{aligned}
\phi_{-}(v)= & \mathbb{E}_{-}\left(\tilde{v}_{\alpha}\right)\left(\frac{e^{\left(m-\tilde{v}_{\alpha}\right) k+\ell_{1} \tilde{v}_{\alpha}} \Psi_{u_{1}^{*}}\left(-\tilde{v}_{\alpha} \sqrt{D_{1}}\right)}{\tilde{v}_{\alpha}\left(\tilde{v}_{\alpha}-m\right)}+\frac{2 e^{m \ell_{1}} \tilde{\Psi}_{u_{1}^{*}}\left(-m \sqrt{D_{1}}\right)}{\tilde{v}_{\alpha}^{2}-m^{2}}\right. \\
& \left.-\frac{e^{\left(m+\tilde{v}_{\alpha}\right) k-\ell_{1} \tilde{v}_{\alpha}} \tilde{\Psi}_{u_{1}^{*}}\left(\tilde{v}_{\alpha} \sqrt{D_{1}}\right)}{\tilde{v}_{\alpha}\left(m+\tilde{v}_{\alpha}\right)}\right), \quad k<\ell_{1},
\end{aligned}
$$

where the constants $u_{1}^{*}$ and $u_{2}^{*}$ are given by

$$
u_{1}^{*}=\frac{\ell_{1}-k}{\sqrt{D_{1}}} \quad \text { and } \quad u_{2}^{*}=\frac{k-\ell_{2}}{\sqrt{D_{2}}},
$$

based on the constant $u^{*}$ in (A.3).

The Fourier transforms we have computed so far are valid in case the stock price process starts between the two barriers. We do not derive transforms for the Greeks, because their transforms are already rather complicated for the single-sided Parisian options as given in [8]. For the double-sided Parisian option the derivation will be very confusing because the Fourier transform is a product of $\mathbb{E}_{ \pm}$and $E\left(c_{1}, c_{2}\right)$, where both terms have dependencies on $\ell_{1}, \ell_{2}$ and $k$. The alternative of numerical differentiation of the inverted Fourier transform delivers accurate Greeks as is shown in the section on numerical examples. The next section treats the case in which the stock price process is already above the upper or below the lower barrier for some time, i.e., the stock price process is "already in the excursion."

\section{Already in the excursion}

Suppose at some time $t_{*}>0$ the stock has already been trading above the level $L_{2}$ for a couple of days and it will only take an extra period of length $d$ for the Parisian 
to knock in. We reset the time to zero and denote the remaining time to expiry by $T$. Now the option knocks in if the stock price process $S$ stays above the level $L_{2}$ longer than the time period $d$. If the stock price process hits the level $L_{2}$ before time $d$ has gone by, the contract knocks in as soon as the stock spends either time $D_{2}$ above $L_{2}$ or time $D_{1}$ below $L_{1}$. So it knocks in if $\tau_{d} \leq T$, where

$$
\tau_{d}=d 1_{\left\{T_{L_{2}}>d\right\}}+\tau 1_{\left\{T_{L_{2}} \leq d\right\}} .
$$

Now the probability we are interested in becomes, for $T>d$,

$$
\mathbb{P}\left[S_{T}>K ; \tau_{d} \leq T\right]=\mathbb{P}\left[S_{T}>K ; T_{L_{2}}>d\right]+\mathbb{P}\left[S_{T}>K ; T_{L_{2}} \leq d ; \tau \leq T\right] .
$$

We remark that in the first probability on the right-hand side we ought to add the constraint that $T>d$, otherwise the Parisian knock-in has not taken place. The reason for leaving this out here is that we know the value of the Parisian knock-in contract to be zero in case of $T<d$, and we should not invert the Fourier transform in this situation. First, we restate the problem in terms of standard Brownian motion and recognize that the stock trading above the level $L_{2}$ translates into the case $\ell_{1}<\ell_{2}<0$. We start calculating the Fourier transform $\phi_{1}$ of the first probability on the right-hand side, and after that we shall compute $\phi_{2}$, the Fourier transform of the second probability on the right-hand side. We want to use the strong Markov property later on, so we have to rewrite the probability and split the Fourier transform into two parts via

$$
\begin{aligned}
\phi_{1}(v) & =\int_{0}^{\infty} e^{(i v-\alpha) T} \mathbb{E}\left[e^{m W_{T}} 1_{\left\{W_{T}>k\right\}}\left(1-1_{\left\{T_{\ell_{2}} \leq d\right\}}\right)\right] \mathrm{d} T \\
& =: \phi_{1,1}(v ; k)-\phi_{1,2}(v ; k),
\end{aligned}
$$

where we explicitly add the $k$ to the notation of $\phi_{1,1}$ and $\phi_{1,2}$ because we want to use $k$ as a parameter. The first part $\phi_{1,1}$ can be computed as

$$
\begin{aligned}
\phi_{1,1}(v ; k) & =\int_{0}^{\infty} e^{(i v-\alpha) T} \frac{1}{\sqrt{2 \pi T}} \int_{k}^{\infty} e^{m x} e^{-\frac{x^{2}}{2 T}} \mathrm{~d} x \mathrm{~d} T \\
& =\frac{1}{\tilde{v}_{\alpha}} \int_{k}^{\infty} e^{m x-|x| \tilde{v}_{\alpha}} \mathrm{d} x= \begin{cases}\frac{e^{\left(m-\tilde{v}_{\alpha}\right) k}}{\tilde{v}_{\alpha}\left(\tilde{v}_{\alpha}-m\right)}, & k \geq 0, \\
\frac{2}{\left(\tilde{v}_{\alpha}^{2}-m^{2}\right)}-\frac{e^{\left(m+\tilde{v}_{\alpha}\right) k}}{\tilde{v}_{\alpha}\left(m+\tilde{v}_{\alpha}\right)}, & k<0 .\end{cases}
\end{aligned}
$$

Here we have used the same type of arguments as in (4.6). Now for $\phi_{1,2}$ we get after conditioning on $\mathcal{F}_{T_{\ell_{2}}}$ and multiple applications of Fubini that

$$
\phi_{1,2}(v ; k)=e^{m \ell_{2}} \mathbb{E}\left[e^{(i v-\alpha) T_{\ell_{2}}} 1_{\left\{T_{\ell_{2}} \leq d\right\}}\right] \int_{0}^{\infty} e^{(i v-\alpha) T} \mathbb{E}\left[e^{m W_{T}} 1_{\left\{W_{T}>k-\ell_{2}\right\}}\right] \mathrm{d} T .
$$

The integral on the right-hand side of this equation equals $\phi_{1,1}\left(v ; k-\ell_{2}\right)$. In Appendix A.2 we compute in (A.6) the expectation on the right-hand side above. Adding the results gives

$$
\phi_{1}(v)=\phi_{1,1}(v ; k)-\left(e^{\ell_{2}\left(m+\tilde{v}_{\alpha}\right)} \mathcal{N}\left(c_{+}^{\text {up }}\right)+e^{\ell_{2}\left(m-\tilde{v}_{\alpha}\right)} \mathcal{N}\left(c_{-}^{\text {up }}\right)\right) \phi_{1,1}\left(v ; k-\ell_{2}\right),
$$


where

$$
c_{ \pm}^{\mathrm{up}}=\frac{\ell_{2} \pm \tilde{v}_{\alpha} d}{\sqrt{d}}
$$

and the superscript $u p$ is used in this notation to denote that the initial stock price is above both barriers. We still have to compute $\phi_{2}$, which we can rewrite by conditioning on $\mathcal{F}_{T_{\ell_{2}}}$. We use the complete, rather elaborate, notation for the Parisian stopping time to explain the strong Markov property in more detail via

$$
\begin{aligned}
\phi_{2}(v) & =\int_{0}^{\infty} e^{(i v-\alpha) T} \mathbb{E}\left[e^{m W_{T}} 1_{\left\{W_{T}>k\right\}} 1_{\left\{T_{\ell_{2}} \leq d\right\}} 1_{\{\tau \leq T\}}\right] \mathrm{d} T \\
& =e^{m \ell_{2}} \int_{0}^{\infty} e^{(i v-\alpha) T} \mathbb{E}\left[1_{\left\{T_{\ell_{2}} \leq d\right\}} \mathbb{E}\left[e^{m W_{\tau}} 1_{\left\{W_{\tau}>k-\ell_{2}\right\}} 1_{\{\tilde{\tau} \leq t\}}\right]_{t=T-T_{\ell_{2}}}\right] \mathrm{d} T,
\end{aligned}
$$

where

$$
\tau=T_{D_{1}, D_{2}}^{\ell_{1}-, \ell_{2}+} \quad \text { and } \quad \tilde{\tau}=T_{D_{1}, D_{2}}^{\left(\ell_{1}-\ell_{2}\right)-, 0+} .
$$

Again, by substitution and multiple Fubini we get an equation for $\phi_{2}$ like (5.1), namely

$$
\phi_{2}(v)=e^{m \ell_{2}} \mathbb{E}\left[e^{(i v-\alpha) T_{\ell_{2}}} 1_{\left\{T_{\ell_{2}} \leq d\right\}}\right] \int_{0}^{\infty} e^{(i v-\alpha) T} \mathbb{E}\left[e^{m W_{T}} 1_{\left\{W_{T}>k-\ell_{2}\right\}} 1_{\{\tilde{\tau} \leq T\}}\right] \mathrm{d} T .
$$

We recognize immediately the first part of $\phi_{1,2}$ given in (5.1) in this equation. The integral on the right-hand side is in fact nothing else than the original problem we are solving, for different barriers. So finally we have

$$
\begin{aligned}
\phi(v)= & \phi_{1,1}(v ; k)+\left(e^{\ell_{2}\left(m+\tilde{v}_{\alpha}\right)} \mathcal{N}\left(c_{+}^{\text {up }}\right)+e^{\ell_{2}\left(m-\tilde{v}_{\alpha}\right)} \mathcal{N}\left(c_{-}^{\text {up }}\right)\right) \\
& \times\left(\phi\left(v ; k-\ell_{2}, \ell_{1}-\ell_{2}, 0\right)-\phi_{1,1}\left(v ; k-\ell_{2}\right)\right), \quad \ell_{1} \leq \ell_{2}<0,
\end{aligned}
$$

where $\phi\left(v ; k-\ell_{2}, \ell_{1}-\ell_{2}, 0\right)$ denotes the Fourier transform as defined in the previous section for the strike $k-\ell_{2}$, lower level $\ell_{1}-\ell_{2}$ and upper level 0 . For the case where the initial stock price is below both levels, i.e., $0<\ell_{1}<\ell_{2}$, we introduce the down version of the $c$ constants as

$$
c_{ \pm}^{\text {down }}=\frac{-\ell_{1} \pm \tilde{v}_{\alpha} d}{\sqrt{d}} .
$$

Following the same steps we can derive the Fourier transform for this case as

$$
\begin{aligned}
\phi(v)= & \phi_{1,1}(v ; k)+\left(e^{\ell_{1}\left(m-\tilde{v}_{\alpha}\right)} \mathcal{N}\left(c_{+}^{\text {down }}\right)+e^{\ell_{1}\left(m+\tilde{v}_{\alpha}\right)} \mathcal{N}\left(c_{-}^{\text {down }}\right)\right) \\
& \times\left(\phi\left(v ; k-\ell_{1}, 0, \ell_{2}-\ell_{1}\right)-\phi_{1,1}\left(v ; k-\ell_{1}\right)\right), \quad 0<\ell_{1} \leq \ell_{2} .
\end{aligned}
$$

Now we are able to price the double-sided Parisian knock-in call for all combinations of initial stock price value, strike and barriers. The next section relates the Fourier transforms computed so far to the double-sided Parisian knock-in put. 


\section{The Parisian put}

For the put option, we need in analogy with (2.3) to calculate the probability

$$
\mathbb{P}_{r}\left[S_{T} \leq K ; T_{D_{1}, D_{2}}^{L_{1}-L_{2}+} \leq T\right]
$$

In [8] the authors use an alternative type of put-call parity. Here we suggest the approach taken in Chap. 11 of [4], i.e., writing the probability as the difference of two probabilities, namely

$$
\mathbb{P}_{r}\left[T_{D_{1}, D_{2}}^{L_{1}-, L_{2}+} \leq T\right]-\mathbb{P}_{r}\left[S_{T} \geq K ; T_{D_{1}, D_{2}}^{L_{1}-, L_{2}+} \leq T\right]
$$

where the second probability is exactly the probability given in (2.3) and the first probability can be obtained by taking the limit of (2.3) for $K \downarrow 0$. So we have to take $k \rightarrow-\infty$ in (4.8) and (4.10), resulting in

$$
\phi(v)=\frac{2 e^{m \ell_{2}} \Psi\left(m \sqrt{D_{2}}\right)}{\tilde{v}_{\alpha}^{2}-m^{2}} \mathbb{E}_{+}\left(\tilde{v}_{\alpha}\right)+\frac{2 e^{m \ell_{1}} \Psi\left(-m \sqrt{D_{1}}\right)}{\tilde{v}_{\alpha}^{2}-m^{2}} \mathbb{E}_{-}\left(\tilde{v}_{\alpha}\right) \quad \text { for } k \rightarrow-\infty
$$

Note that we have used the equation for $\phi$ in which we assume that $L_{1} \leq S_{0} \leq L_{2}$. Prices for the Parisian put option for the case where it is already in the excursion can be obtained by taking $k \rightarrow-\infty$ in the formulas for $\phi$ as given in Sect. 5. Now that we have Fourier transforms for double-sided Parisian knock-in options, both put and call, we continue discussing the other types of Parisian contract types that we can construct from the double-sided Parisian contract.

\section{Other types of Parisian contracts}

Recall the way we defined $\phi_{+}$and $\phi_{-}$in (4.2), where $\phi_{+}$is the Fourier transform with respect to the parameter $T$ of the probability of the intersection of the following events:

- the event that the stock price at time $T$ exceeds the strike price;

- the event $\left\{\tau^{+} \leq T\right\}$, which states that the stock price made an excursion of length $D_{2}$ above the level $L_{2}$ at some time before $T$;

- the event $\left\{\tau^{+}<\tau^{-}\right\}$, which represents the stock price process paths that spend time $D_{2}$ above the level $L_{2}$ before spending time $D_{1}$ below the level $L_{1}$.

For $\phi_{-}$the last two events are replaced by $\left\{\tau^{-} \leq T\right\}$ and $\left\{\tau^{-}<\tau^{+}\right\}$respectively, with analogous interpretation. Now we can construct different types of contracts, like the single-sided Parisian option, by different selections of $\phi_{+}, \phi_{-}$and parameter sets in the following way:

- Type 1. $\phi=\phi_{+}+\phi_{-}$: The double-sided Parisian contract that is paying off when $S$ stays longer than consecutive time $D_{1}$ below the level $L_{1}$ or $D_{2}$ above the level $L_{2}$. 
- Type $2 a . \phi=\phi_{+}+\phi_{-}$and $\ell_{1} \rightarrow-\infty$ or $D_{1} \rightarrow \infty$ : The single-sided Parisian upand-in call. Taking these limits in the equations for $\phi$ gives the formulas in [8], where we remark that we compute transforms of the probabilities needed to calculate the Parisian option value, whereas the authors of [8] compute transforms of the non-discounted payoff.

- Type 2b. $\phi=\phi_{+}+\phi_{-}$and $\ell_{2} \rightarrow \infty$ or $D_{2} \rightarrow \infty$ : Analogously to the previous case, this is the single-sided down-and-in call.

- Type 3a. $\phi=\phi_{+}$: The Parisian contract that pays off when $S$ stays above the level $L_{2}$ for a consecutive period of length $D_{2}$, without having been below $L_{1}$ for a period $D_{1}$ before. This contract type is called the double-sided Parisian up-beforedown-in call.

- Type 3b. $\phi=\phi_{-}$: The Parisian contract that pays off when $S$ stays below the level $L_{1}$ for a consecutive period of length $D_{1}$, without having been above $L_{2}$ for a period $D_{2}$ before, called the double-sided Parisian down-before-up-in call.

A very rough upper bound for the prices of the specified contract types is the plain-vanilla call. A less rough upper bound is given by the double-sided knock-in barrier call. We continue discussing the price ordering of the contracts above. For double-sided barriers this has already been done in [16]. We remark that for given levels $L_{1}, L_{2}$ and periods $D_{1}, D_{2}$ the contract type 1 is strictly most expensive, because given one of the contract types $2 \mathrm{a}, 2 \mathrm{~b}, 3 \mathrm{a}$ or $3 \mathrm{~b}$ there exist stock price paths that do trigger contract type 1 without knocking in the given contract type. Less expensive are the single-sided Parisian options given by the contract types $2 \mathrm{a}$ and $2 \mathrm{~b}$. For the one-sided Parisians there is only one level for the stock price process that can cause a knock-in of the contract. However, the behavior of the stock price above the other level cannot cause the Parisian option to knock out, which is the case for the cheapest contract types $3 \mathrm{a}$ and $3 \mathrm{~b}$. This type of double-sided Parisians does not only have just one stock price level that can cause a knock-in, but it also contains another level for the stock price process that knocks out the contract if the stock price process spends a certain time above or below this level. Note that it is possible to obtain (numerical) values for the single-sided Parisian contract without actually taking the limits as proposed in the description of types $2 \mathrm{a}$ and $2 \mathrm{~b}$. For a given time to expiry $T$ the value of the single-sided Parisian down-and-in call can be obtained by inverting the Fourier transform of the double-sided Parisian contract for some $L_{2}$ and $D_{2}>T$. Similarly we get the value of the single-sided up-and-in by inverting the transform for some $L_{1}$ and $D_{1}>T$. We shall illustrate these remarks in the section on numerical examples. Figure 1 shows the relations between the double-sided and single-sided Parisian contracts, where we abbreviate the double-sided Parisian in-call by DPIC and the out call by DPOC. The single-sided contracts are either up (P.U.) or down (P.D.) and either in (P.I.) or out (P.O.) contracts. The same type of scheme could be drawn for the Parisian put contracts. Now that we have computed and discussed various types of Parisian contracts, we discuss the Fourier inversion in the next section. 


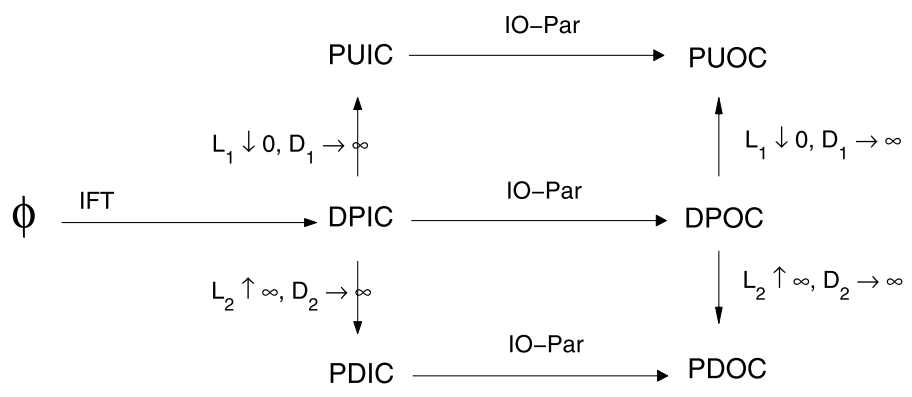

Fig. 1 Relations between different types of Parisian contracts

\section{Fourier inversion algorithm}

\subsection{General Fourier inversion}

Apart from deriving Fourier transforms for the relevant probabilities for the doublesided Parisian option contracts, we are also interested in numerical values for these options. We have to obtain these values by inversion of the Fourier transform for the probabilities we need to construct the contract, where we recall formula (2.2). In (4.1), the definition of $\phi$, the Fourier transform of the probability $P_{r}(T)$ is stated and values for $P_{r}(T)$ can be obtained by the standard Fourier inversion formula

$$
P_{r}(T)=\frac{e^{a T}}{2 \pi} \int_{-\infty}^{\infty} e^{i v T} \phi(v) \mathrm{d} v=\frac{2 e^{a T}}{\pi} \int_{0}^{\infty} \cos (v T) \Re\{\phi(v)\} \mathrm{d} v,
$$

where $\mathfrak{R}\{\phi(v)\}$ denotes the real part of $\phi(v)$. We recall that $a$ is a damping factor to ensure integrability in (4.1) and that $\alpha$ we are using in all the derivations is given by $\alpha=a-\frac{1}{2} m^{2}$, where $m$ is a constant coming from the Girsanov transform, implicitly defined in (2.4). To arrive at the integral over the positive real line on the right-hand side, we refer to [2], where the key idea is that from $P_{r}(T)$ with domain on the positive real line a symmetric function $f$ on the whole real line can be constructed by $f(t):=P_{r}(|t|)$. Now it is straightforward to obtain numerical values for $P_{r}$ by discretizing and truncating the integral, i.e.,

$$
P_{r}(T)=\frac{2 h e^{a T}}{\pi} \sum_{j=0}^{N} \cos \left(v_{j} T\right) \Re\left\{\phi\left(v_{j}\right)\right\}+\epsilon_{t}+\epsilon_{d},
$$

where $h$ is the step size, $N h$ the level of truncation and $\epsilon_{d}$ and $\epsilon_{t}$ respectively the discretization and the truncation error. Following [2], we analyze the discretization error $\epsilon_{d}$ using the Poisson summation formula which assumes an Euler approximation for the integral in (8.1). In Appendix A.4 we derive for the discretization error that

$$
\epsilon_{d} \leq \frac{2 e^{-a\left(\frac{2 \pi}{h}-T\right)}}{1-e^{-\alpha \frac{2 \pi}{h T}}} \quad \text { for } h<\frac{2 \pi}{T} .
$$


In Appendix A.5 we analyze the truncation error and find that

$$
\epsilon_{t} \leq \arctan \left(\frac{a}{h(N-1)}\right)
$$

which is going to zero very slowly. Experiments show that the number of terms needed for a given accuracy is much less than indicated by the bound on $\epsilon_{t}$. This suggests that the bound presented in (8.2) is not strong enough. The next section elaborates on the truncation error bound in order to come up with a better estimate for the accuracy.

\subsection{Remarks on the truncation bound}

The $\arctan (\cdot)$-term in (8.2) is going to zero very slowly, resulting in an impractical number of terms we have to compute in the truncated sum of (8.1) in case we want to obtain a reasonable theoretical error of e.g. $1 \%$. We could try to solve this by using instead of the standard FFT the alternative inversion algorithms as Euler summation, which is proposed by [2], or approximation of the Fourier transform by polynomiallike functions of which the inverse is known, as has been done by [3]. Here we introduce a slightly modified version of the Euler summation, the average summation, because it seems to fit better to the limiting properties of the Fourier transform; but it has the same drawback as the other methods, which is that it is not possible to give a reasonable bound for the truncation error without using heuristics. In [1] the authors conclude that it is often difficult to provide reasonable error bounds and therefore they suggest to use two "good" methods and compare the results for an error estimate. By numerically studying the behavior of the sum in (8.1) it appears that this sum as a function of $N$ oscillates for $N$ big enough around its limiting value with a double frequency, namely a high frequency determined by $T$ and a low frequency determined by $D$, which is the smallest number divisible by both $D_{1}$ and $D_{2}$. As argued in Sect. 7, the Fourier transform of the single-sided Parisian is given by that of the double-sided Parisian for one of $D_{1,2}$ going to infinity. For this single-sided Fourier transform one can actually show, by using a Taylor expansion of the Fourier transform, that it exhibits this oscillating behavior. For the double-sided Parisian, the Fourier transform is a sum and a product of functions oscillating with a high frequency $T$ and low frequencies $D_{1}$ and $D_{2}$, and therefore the transform itself oscillates with a low frequency in which products and sums of frequencies determined by $D_{1}$ and $D_{2}$ fit, i.e., a frequency determined by period $D$, the smallest number divisible by both $D_{1}$ and $D_{2}$. In order to get rid of the high-frequency oscillation, we propose to average the sum over the last $M$ terms as

$$
\sum_{j=0}^{\infty} \cos \left(v_{j} T\right) \Re\left\{\phi\left(v_{j}\right)\right\} \approx S_{N, M}:=\frac{1}{M} \sum_{k=1}^{M} S_{N+k}, \quad \text { where } M=\frac{2 \pi}{D h},
$$

where $S_{N}$ is the partial sum of the first $N$ terms. Now, for $n$ large enough, also the partial averaged sums $S_{n, M}$ will oscillate around the limiting value but with a much smaller amplitude. In the following algorithm we use this oscillating behavior 
to come up with an estimate for the error, because we know that the local maxima (and minima) are all above (respectively below) the limit we are interested in. If $y$ is an $N$-dimensional vector containing the partial averaged sums, i.e., $y[k]=S_{k, M}$ for $0 \leq k \leq N$, the local extrema are defined by

$$
\begin{aligned}
& y[i] \text { is a local max } \Longleftrightarrow y[i-1]<y[i]>y[i+1], \\
& y[i] \text { is a local min } \Longleftrightarrow y[i-1]>y[i]<y[i+1] \text {, }
\end{aligned}
$$

for $i \in\{1, \ldots, N-1\}$. The inversion algorithm now becomes

$-n=2, y[0]=0, y[1]=1$

- Choose $\alpha$ and $h$ such that the discretization error is $\epsilon_{d}$

- Determine $M$

- Repeat

$$
\begin{aligned}
& y[n]=S_{n, M} \\
& P_{\max }=\min \{y[i], 0 \leq i \leq n \mid y[i] \text { is a local max }\} \\
& P_{\min }=\max \{y[i], 0 \leq i \leq n \mid y[i] \text { is a local min }\}
\end{aligned}
$$

- Until $P_{\max }-P_{\min }<2 \epsilon_{d}$

- $P=\left(P_{\max }+P_{\min }\right) / 2$ with truncation error $\epsilon_{t}<\epsilon_{d}$.

Experiments show that by using this algorithm we obtain a given accuracy much more quickly than the truncation bound in (8.2) suggests. They even show that averaging over $M$ terms diminishes the amplitude of the sum drastically compared both to plain summation and Euler summation. As we are now able to obtain numerical values from the Fourier transforms we previously calculated, we conclude the paper with a section on numerical examples.

\section{Numerical examples}

In this section we illustrate the inversion of the Parisian option prices. Throughout the examples we use, if not mentioned otherwise, $S_{0}=100, K=100, L_{1}=90$, $L_{2}=110, D_{1}=D_{2}=10 / 250, T=1, r=3.5 \%, \sigma=25 \%$ and $h=0.2$, which for $\alpha \geq 0.65$ will give a discretization error less than $10^{-8}$. If we denote quantities in units of (business) days, then $n$ days correspond to $n / 250$ years. We use Fourier inversion as described in the previous section and obtain prices typically within one second. Simulation of Parisian options usually is much slower, on the order of minutes for the same accuracy, because one needs to choose a really fine grid in order to capture the local behavior of the underlying Brownian motion.

\subsection{Double-sided Parisian prices}

In this example we show how the double-sided Parisian knock-in option price behaves. First we vary the time that should be spent by the stock price process below or above the respective barriers $L_{1}$ and $L_{2}$. 

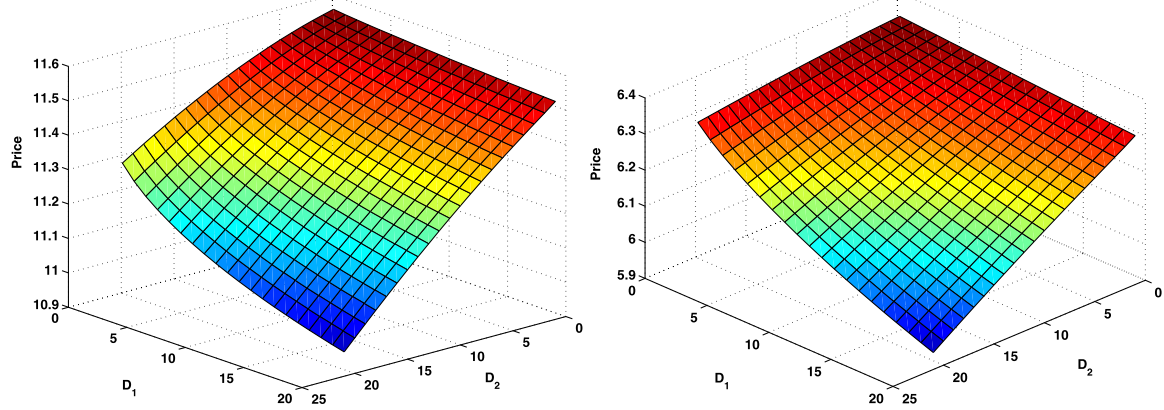

Fig. 2 Double-sided Parisian knock-in call option prices for various times $D_{1}$ and $D_{2}$ with $S_{0}=100$ (left) and $S_{0}=90$ (right)

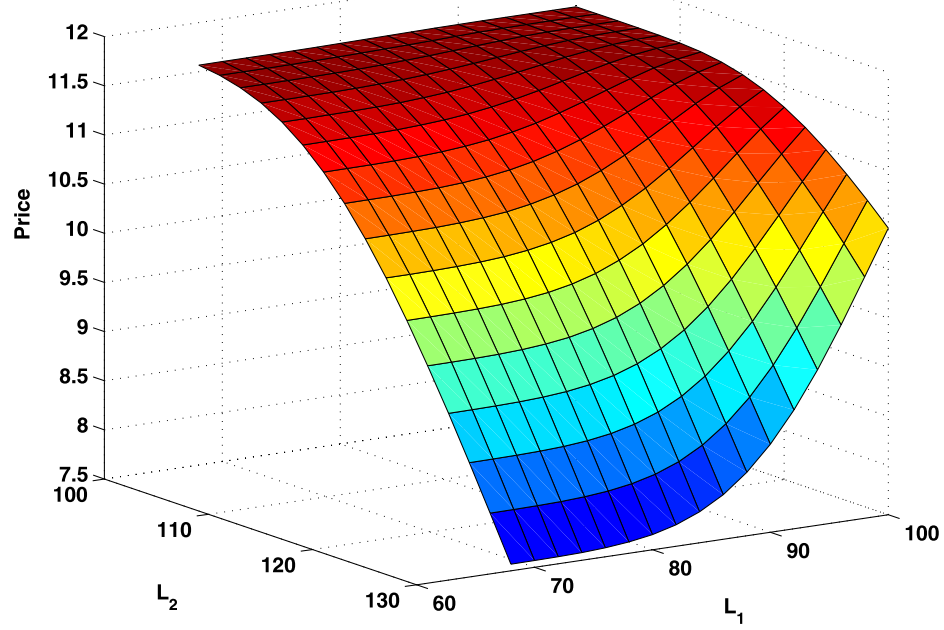

Fig. 3 Double-sided Parisian knock-in call option prices for various barriers $L_{1}$ and $L_{2}$ with $S_{0}=100$

Figure 2 shows that the prices of the Parisian knock-in call decrease as the times $D_{1}$ to spend below $L_{1}$ or $D_{2}$ to spend above $L_{2}$ increase. The graph on the lefthand side shows that in case the stock price is exactly between the levels $L_{1}$ and $L_{2}$, the time $D_{2}$ has more influence on the price than $D_{1}$. This is a result of the fact that knocking in via the upper level and ending up above the strike is more likely than knocking in via the lower level and ending up above the strike. In the righthand graph we changed the initial stock price to $S_{0}=90$. As we are now more in the neighborhood of the lower level $L_{1}$, the influence of $D_{1}$ in comparison to $D_{2}$ increases, which can clearly be seen in the graph.

Instead of computing pricing for various $D_{1}$ and $D_{2}$, we can also vary the barriers $L_{1}$ and $L_{2}$. The graph in Fig. 3 shows the behavior of the double-sided Parisian knock-in call for different levels. Just like in the previous example, the Parisian option price is much more sensitive to the upper level $L_{2}$ than to the lower level $L_{1}$. 

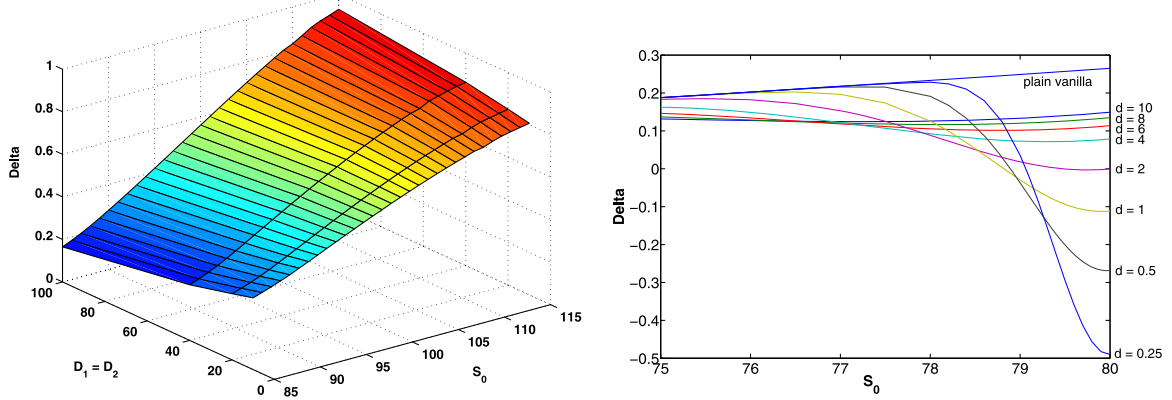

Fig. 4 Double-sided Parisian knock-in call option deltas for various times $D_{1}=D_{2}$ and $S_{0}$ (left) and for various $d$ and $S_{0}$ in case the barriers are set to $L_{1}=80$ and $L_{2}=120$ (right)

\subsection{Delta}

As mentioned at the end of Sect. 4 the most practical way to obtain the Greeks is by numerical differentiation. Here we give an example of the delta, the partial derivative of the double-sided Parisian knock-in call price with respect to the initial stock price value $S_{0}$. The left-hand side surface plot in Fig. 4 shows the delta for different times $D_{1}=D_{2}$ that the stock price should spend below $L_{1}$ or above $L_{2}$ to knock in. The notation on the $D_{1}=D_{2}$-axis is in days. The surface plot shows that increasing periods $D$ sharpen the shape of the delta curve with respect to the initial stock price value.

The delta in the surface plot looks still quite similar to the plain-vanilla delta. For example the gamma, i.e., the partial derivative of the delta with respect to the stock price, seems to be always positive. More spectacular behavior of the delta can be observed if we widen the barriers to $L_{1}=80$ and $L_{2}=120$ and look at the region $S_{0} \leq L_{1}$. In that case we need the formulas from Sect. 5 , because the stock is already in the excursion. Recall that $d$ denotes the time that the stock price process still has to stay below $L_{1}$ before the Parisian actually knocks in. The right panel of Fig. 4 shows how delta behaves for different $d$. The interesting case is the case for which $d$ approaches zero. In this case the stock has stayed below the barrier for almost long enough to knock the Parisian in. If, at this time, the stock is far away from the barrier, the Parisian will knock in almost surely and the delta resembles the plainvanilla delta, e.g. the $d=0.25$ line for $S_{0}<77$. However, if the stock is close to the barrier, an increase of the stock price will decrease the probability of knock-in. So, an increase of the stock price will decrease the price of the Parisian option, which results in both negative gamma and delta as can be seen on the $d=0.25$ line for $S_{0}>79$.

\subsection{Various contract types}

In this numerical example we compare the prices of the double-sided Parisian contract types as listed in Sect. 7. We add the double-sided barrier option to this comparison and we implement the method described in [20] to obtain the prices. Table 1 contains prices of the various contracts for different values of the initial stock price. 
Table 1 Prices of various option contracts

\begin{tabular}{llllll}
\hline Contract type & $S_{0}=90$ & $S_{0}=95$ & $S_{0}=100$ & $S_{0}=105$ & $S_{0}=110$ \\
\hline Plain vanilla & 6.362 & 8.768 & 11.591 & 14.800 & 18.349 \\
Double-sided knock-in & 6.362 & 8.767 & 11.591 & 14.799 & 18.349 \\
Double-sided $P$ knock-in (1) & 6.236 & 8.568 & 11.371 & 14.608 & 18.226 \\
Single $P$ up-in (2a) & 5.792 & 8.218 & 11.113 & 14.435 & 18.129 \\
P up-before-down-in (3a) & 3.568 & 6.844 & 10.284 & 13.957 & 17.886 \\
Single $P$ down-in (2b) & 2.676 & 1.742 & 1.123 & 0.719 & 0.457 \\
$P$ down-before-up-in (3b) & 2.668 & 1.723 & 1.087 & 0.651 & 0.339 \\
\hline
\end{tabular}

The numbers refer to the contract type numbers in their description in Sect. 7 and $P$ denotes Parisian. The plain-vanilla call is the most expensive contract, very closely followed by the double-sided knock-in call barrier option. The double-sided Parisian knock-in call is again cheaper than its standard version, where the price difference is determined by $D_{1}$ and $D_{2}$ as is shown in Fig. 2. Both the single-sided Parisian contracts are cheaper than the double-sided in version, whereas the double-sided upbefore-down-in and down-before-up-in contracts are again cheaper than the singlesided up-and-in and down-and-in contracts respectively.

\subsection{Theta in the tails}

In this example we consider the evolution in time of the double-sided Parisian knockin call option for values of the initial stock price $S_{0}$ below the lower barrier $L_{1}$ or above the upper barrier $L_{2}$, which we denote by the lower and upper tail respectively. We need to invert the Fourier transform as given in Sect. 5, which treats the case that the stock price process is already in the excursion. The partial derivative of the option price with respect to the time to maturity is called the theta. The price of plain-vanilla call options on non-dividend paying stocks always decreases as the time to expiry decreases. In practice traders with a long position in an option try to make a profit from trading their hedging portfolio. This profit should compensate the theta they are losing in the option's long position. We start calculating prices of options that expire in $T=1$ year. Then we let time run in steps of one day, so both the time to expiry and the remaining time the Parisian option needs to stay away from the barrier decrease by one day. In Table 2 we give this price evolution for $S_{0} \leq L_{1}$ and the barriers $L_{1}=80, L_{2}=120$, where $d$ is printed in days. The columns Par and Plain contain the Parisian and plain-vanilla option values respectively. The table shows four cases which demonstrate how the increase in option price value caused by the increase of the probability to knock in competes with the time-decay of the option:

- The case $S_{0}=80$. In this case the excursion is about to start afresh and the decrease of the option price shown in the table is a result of the time-decay of the Parisian option.

- The case $S_{0}=76$. In this case the stock is actually in the excursion and each day the increase in value caused by the probability to knock in exceeds the decrease in 
Table 2 Price evolution for various values of $S_{0} \leq L_{1}, L_{1}=80$ and $L_{2}=120$

\begin{tabular}{|c|c|c|c|c|c|c|c|c|}
\hline \multirow[t]{2}{*}{$d$} & \multicolumn{2}{|c|}{$S_{0}=70$} & \multicolumn{2}{|c|}{$S_{0}=74$} & \multicolumn{2}{|c|}{$S_{0}=76$} & \multicolumn{2}{|c|}{$S_{0}=80$} \\
\hline & Par & Plain & Par & Plain & Par & Plain & Par & Plain \\
\hline 10 & 0.953 & 0.957 & 1.474 & 1.547 & 1.737 & 1.923 & 2.257 & 2.856 \\
\hline 9 & 0.946 & 0.949 & 1.474 & 1.536 & 1.740 & 1.911 & 2.240 & 2.841 \\
\hline 8 & 0.939 & 0.941 & 1.475 & 1.525 & 1.744 & 1.898 & 2.222 & 2.825 \\
\hline 7 & 0.932 & 0.933 & 1.476 & 1.514 & 1.750 & 1.885 & 2.205 & 2.809 \\
\hline 6 & 0.925 & 0.925 & 1.476 & 1.503 & 1.759 & 1.873 & 2.187 & 2.794 \\
\hline 5 & 0.917 & 0.917 & 1.475 & 1.492 & 1.770 & 1.860 & 2.170 & 2.778 \\
\hline 4 & 0.909 & 0.909 & 1.472 & 1.481 & 1.783 & 1.848 & 2.152 & 2.762 \\
\hline 3 & 0.901 & 0.901 & 1.467 & 1.470 & 1.797 & 1.835 & 2.135 & 2.747 \\
\hline 2 & 0.893 & 0.893 & 1.459 & 1.459 & 1.809 & 1.823 & 2.117 & 2.731 \\
\hline 1 & 0.885 & 0.885 & 1.448 & 1.448 & 1.809 & 1.810 & 2.100 & 2.715 \\
\hline
\end{tabular}

Table 3 Price evolution for various values of $S_{0} \geq L_{2}, L_{1}=80$ and $L_{2}=120$

\begin{tabular}{|c|c|c|c|c|c|c|c|c|}
\hline \multirow[t]{2}{*}{$d$} & \multicolumn{2}{|c|}{$S_{0}=120$} & \multicolumn{2}{|c|}{$S_{0}=122$} & \multicolumn{2}{|c|}{$S_{0}=124$} & \multicolumn{2}{|c|}{$S_{0}=126$} \\
\hline & Par & Plain & Par & Plain & Par & Plain & Par & Plain \\
\hline 10 & 25.57 & 26.28 & 27.43 & 27.98 & 29.33 & 29.70 & 31.21 & 31.46 \\
\hline 9 & 25.54 & 26.26 & 27.42 & 27.95 & 29.32 & 29.68 & 31.21 & 31.43 \\
\hline 8 & 25.51 & 26.23 & 27.40 & 27.93 & 29.31 & 29.66 & 31.21 & 31.41 \\
\hline 7 & 25.48 & 26.21 & 27.39 & 27.91 & 29.31 & 29.63 & 31.21 & 31.39 \\
\hline 6 & 25.45 & 26.18 & 27.38 & 27.88 & 29.31 & 29.61 & 31.21 & 31.36 \\
\hline 5 & 25.42 & 26.16 & 27.37 & 27.86 & 29.32 & 29.58 & 31.21 & 31.34 \\
\hline 4 & 25.39 & 26.13 & 27.38 & 27.83 & 29.33 & 29.56 & 31.22 & 31.32 \\
\hline 3 & 25.36 & 26.11 & 27.39 & 27.81 & 29.36 & 29.54 & 31.24 & 31.29 \\
\hline 2 & 25.33 & 26.08 & 27.43 & 27.78 & 29.40 & 29.51 & 31.25 & 31.27 \\
\hline 1 & 25.30 & 26.06 & 27.53 & 27.76 & 29.46 & 29.49 & 31.24 & 31.25 \\
\hline
\end{tabular}

value from the time-decay and there is a monotonic increase of the Parisian price to the plain-vanilla price.

- The case $S_{0}=74$. This is a kind of intermediate case, as the increase in value due to the increase in knock-in probability is almost canceled by the decrease in value due to the time decay.

- The case $S_{0}=70$. In this case the option is very sure to be knocked in. The price almost resembles the plain-vanilla price and the very small increase in probability of knock-in is not enough to compensate for the time-decay. The Parisian theta is almost equal to the plain-vanilla theta.

Table 3 shows that also in the upper tail theta can become negative. The effect is not as strong as in the lower tail, because the probability of the intersection of ending up above the strike and knocking in via the upper tail is much bigger than ending up 
Table 4 Price evolution for various values of $S_{0} \leq L_{1}, L_{1}=90$ and $L_{2}=110$

\begin{tabular}{|c|c|c|c|c|c|c|c|c|}
\hline \multirow[t]{2}{*}{$d$} & \multicolumn{2}{|c|}{$S_{0}=84$} & \multicolumn{2}{|c|}{$S_{0}=86$} & \multicolumn{2}{|c|}{$S_{0}=88$} & \multicolumn{2}{|c|}{$S_{0}=90$} \\
\hline & Par & Plain & Par & Plain & Par & Plain & Par & Plain \\
\hline 10 & 4.028 & 4.051 & 4.702 & 4.751 & 5.436 & 5.521 & 6.236 & 6.362 \\
\hline 9 & 4.012 & 4.032 & 4.686 & 4.731 & 5.416 & 5.500 & 6.212 & 6.340 \\
\hline 8 & 3.997 & 4.014 & 4.669 & 4.711 & 5.397 & 5.479 & 6.188 & 6.317 \\
\hline 7 & 3.981 & 3.995 & 4.653 & 4.691 & 5.378 & 5.457 & 6.164 & 6.295 \\
\hline 6 & 3.966 & 3.976 & 4.638 & 4.671 & 5.360 & 5.436 & 6.140 & 6.272 \\
\hline 5 & 3.950 & 3.958 & 4.623 & 4.651 & 5.343 & 5.415 & 6.116 & 6.250 \\
\hline 4 & 3.935 & 3.939 & 4.610 & 4.631 & 5.328 & 5.394 & 6.092 & 6.227 \\
\hline 3 & 3.919 & 3.920 & 4.597 & 4.611 & 5.315 & 5.372 & 6.068 & 6.205 \\
\hline 2 & 3.901 & 3.902 & 4.585 & 4.591 & 5.307 & 5.351 & 6.044 & 6.182 \\
\hline 1 & 3.883 & 3.883 & 4.570 & 4.571 & 5.308 & 5.329 & 6.019 & 6.160 \\
\hline
\end{tabular}

Table 5 Price evolution for various values of $S_{0} \geq L_{2}, L_{1}=90$ and $L_{2}=110$

\begin{tabular}{|c|c|c|c|c|c|c|c|c|}
\hline \multirow[t]{2}{*}{$d$} & \multicolumn{2}{|c|}{$S_{0}=110$} & \multicolumn{2}{|c|}{$S_{0}=112$} & \multicolumn{2}{|c|}{$S_{0}=114$} & \multicolumn{2}{|c|}{$S_{0}=116$} \\
\hline & Par & Plain & Par & Plain & Par & Plain & Par & Plain \\
\hline 10 & 18.23 & 18.35 & 19.76 & 19.85 & 21.34 & 21.40 & 22.95 & 22.99 \\
\hline 9 & 18.20 & 18.32 & 19.73 & 19.83 & 21.32 & 21.38 & 22.93 & 22.97 \\
\hline 8 & 18.17 & 18.30 & 19.71 & 19.80 & 21.29 & 21.35 & 22.91 & 22.94 \\
\hline 7 & 18.14 & 18.27 & 19.68 & 19.77 & 21.27 & 21.32 & 22.89 & 22.91 \\
\hline 6 & 18.11 & 18.24 & 19.66 & 19.75 & 21.25 & 21.30 & 22.87 & 22.89 \\
\hline 5 & 18.08 & 18.21 & 19.64 & 19.72 & 21.23 & 21.27 & 22.84 & 22.86 \\
\hline 4 & 18.05 & 18.19 & 19.61 & 19.69 & 21.21 & 21.25 & 22.82 & 22.84 \\
\hline 3 & 18.03 & 18.16 & 19.59 & 19.67 & 21.19 & 21.22 & 22.80 & 22.81 \\
\hline 2 & 18.00 & 18.13 & 19.58 & 19.64 & 21.18 & 21.19 & 22.78 & 22.79 \\
\hline 1 & 17.97 & 18.11 & 19.58 & 19.61 & 21.16 & 21.16 & 22.76 & 22.76 \\
\hline
\end{tabular}

above the strike and knocking in via the lower tail. Therefore the Parisian knock-in call will behave more like a plain vanilla for $S_{0}>L_{2}$ than it does for $S_{0}<L_{1}$.

Tables 4 and 5 contain the same information as Tables 2 and 3, except that the Parisian prices are now calculated for narrower barriers $L_{1}=90$ and $L_{2}=110$. The tables show that the negative theta effect disappears in this case. The prices of both the Parisian knock-in call option and the plain-vanilla call decrease as time goes by. The Parisian is very likely to knock in for these narrow barriers and therefore it behaves much more like the plain-vanilla call.

\section{Conclusion}

In this paper we derived the Fourier transform for the probabilities related to the double-sided Parisian in-options. These Parisian options are triggered by a double- 
sided Parisian stopping time. In order to get the Fourier transforms of the options, we derived the Laplace transform of the Parisian stopping time, for which we used excursion theory and we obtained a remarkable result for standard Brownian motion as a corollary. We also treated the case that the Parisian option already has spent some time in the excursion. The Fourier transform of the double-sided Parisian in-call could also be used to obtain the Fourier transform of the equivalent Parisian put. Furthermore, we could derive the single-sided Parisian contract types from the double-sided Parisian as well as a down-before-up contract type. The numerical example treats the behavior of the double-sided Parisian knock-in call contract. In order to obtain actual values, we use average summation as inversion technique. Apart from the prices, we show that the Greeks can be behave rather peculiarly, in the sense that the Parisian contract can have a negative gamma and theta.

Acknowledgements The authors would like to thank M. Jeanblanc for iteratively commenting on the results of Sect. 2, which, finally, resulted in the present text.

Open Access This article is distributed under the terms of the Creative Commons Attribution Noncommercial License which permits any noncommercial use, distribution, and reproduction in any medium, provided the original author(s) and source are credited.

\section{Appendix A}

A.1 Calculation of $\phi_{+}$for $k>\ell_{2}$ and $\phi_{-}$for $k<\ell_{1}$

Here we derive the expressions for $\phi_{+}$and $\phi_{-}$as given by (4.9) and (4.10). The first step is to come up with a formula for $E\left(c_{1}, c_{2}\right)$ for the cases not covered by Lemma 4.2, which is done in the following lemma. Equations (4.9) and (4.10) then follow as a corollary from this lemma in the same way as Corollary 4.3 follows from Lemma 4.2.

Lemma A.1 Let $E\left(c_{1}, c_{2}\right)$ be defined by (4.5) in Lemma 4.2 for the real numbers $c_{1}$ and $c_{2}$. Then the following formulas hold:

$$
\begin{aligned}
E\left(c_{1}, c_{2}\right)= & \frac{2 e^{m c_{1}}}{\tilde{v}_{\alpha}^{2}-m^{2}} \mathbb{E}\left[e^{c_{2} m N} 1_{\left\{N \leq u^{*}\right\}}\right]-\frac{e^{\left(m+\tilde{v}_{\alpha}\right) k-\tilde{v}_{\alpha} c_{1}}}{\tilde{v}_{\alpha}\left(\tilde{v}_{\alpha}+m\right)} \mathbb{E}\left[e^{-c_{2} \tilde{v}_{\alpha} N} 1_{\left\{N \leq u^{*}\right\}}\right] \\
& +\frac{e^{\left(m-\tilde{v}_{\alpha}\right) k+\tilde{v}_{\alpha} c_{1}}}{\tilde{v}_{\alpha}\left(\tilde{v}_{\alpha}-m\right)} \mathbb{E}\left[e^{c_{2} \tilde{v}_{\alpha} N} 1_{\left\{N \geq u^{*}\right\}}\right], \quad k<c_{1}, c_{2}<0,
\end{aligned}
$$

and

$$
\begin{aligned}
E\left(c_{1}, c_{2}\right)= & \frac{e^{\left(m-\tilde{v}_{\alpha}\right) k+\tilde{v}_{\alpha} c_{1}}}{\tilde{v}_{\alpha}\left(\tilde{v}_{\alpha}-m\right)} \mathbb{E}\left[e^{c_{2} \tilde{v}_{\alpha} N} 1_{\left\{N \leq u^{*}\right\}}\right]+\frac{2 e^{m c_{1}}}{\tilde{v}_{\alpha}^{2}-m^{2}} \mathbb{E}\left[e^{c_{2} m N} 1_{\left\{N \geq u^{*}\right\}}\right] \\
& -\frac{e^{\left(m+\tilde{v}_{\alpha}\right) k-\tilde{v}_{\alpha} c_{1}}}{\tilde{v}_{\alpha}\left(\tilde{v}_{\alpha}+m\right)} \mathbb{E}\left[e^{-c_{2} \tilde{v}_{\alpha} N} 1_{\left\{N \geq u^{*}\right\}}\right], \quad k>c_{1}, \quad c_{2}>0,
\end{aligned}
$$


where

$$
u^{*}=\frac{k-c_{1}}{c_{2}} \text {. }
$$

Proof The starting point is (4.7) in Lemma 4.2, which we can write as

$$
E\left(c_{1}, c_{2}\right)=\frac{e^{m c_{1}}}{\tilde{v}_{\alpha}} \int_{0}^{\infty} u e^{-\frac{u^{2}}{2}+c_{2} m u} I_{k-c_{1}-c_{2} u} \mathrm{~d} u,
$$

where $I_{a}$ for real $a$ is given by

$$
I_{a}=\int_{a}^{\infty} e^{m x-|x| \tilde{v}_{\alpha}} \mathrm{d} x= \begin{cases}\frac{e^{\left(m-\tilde{v}_{\alpha}\right) a}}{\tilde{v}_{\alpha}-m}, & a \geq 0, \\ \frac{2 \tilde{v}_{\alpha}}{\tilde{v}_{\alpha}^{2}-m^{2}}-\frac{e^{\left(m+\tilde{v}_{\alpha}\right) a}}{m+\tilde{v}_{\alpha}}, & a<0 .\end{cases}
$$

Now we split the integral in (A.4) into two parts, separated by $u^{*}$ given by (A.3), resulting in

$$
\begin{aligned}
E\left(c_{1}, c_{2}\right)= & \frac{e^{m c_{1}}}{\tilde{v}_{\alpha}}\left(\int_{0}^{u^{*}} u e^{-\frac{u^{2}}{2}+c_{2} m u} I_{k-c_{1}-c_{2} u} \mathrm{~d} u\right. \\
& \left.+\int_{u^{*}}^{\infty} u e^{-\frac{u^{2}}{2}+c_{2} m u} I_{k-c_{1}-c_{2} u} \mathrm{~d} u\right) .
\end{aligned}
$$

For the case $k<c_{1}$ and $c_{2}<0$, the subscript of $I$ is negative for the first integral and positive for the second integral, giving

$$
\begin{aligned}
E\left(c_{1}, c_{2}\right)= & \frac{e^{m c_{1}}}{\tilde{v}_{\alpha}}\left(\int_{0}^{u^{*}} u e^{-\frac{u^{2}}{2}+c_{2} m u}\left(\frac{2 \tilde{v}_{\alpha}}{\tilde{v}_{\alpha}^{2}-m^{2}}-\frac{e^{\left(m+\tilde{v}_{\alpha}\right)\left(k-c_{1}-c_{2} u\right)}}{\tilde{v}_{\alpha}+m}\right) \mathrm{d} u\right. \\
& \left.+\int_{u^{*}}^{\infty} u e^{-\frac{u^{2}}{2}+c_{2} m u} \frac{e^{\left(m+\tilde{v}_{\alpha}\right)\left(k-c_{1}-c_{2} u\right)}}{\tilde{v}_{\alpha}-m} \mathrm{~d} u\right) .
\end{aligned}
$$

Now put in this equation every non- $u$ term in front of the integral and rewrite the remaining integral as an expectation using the density of $N$ given in (3.1) to arrive at (A.1). The case $k>c_{1}$ and $c_{2}>0$ is analogous, except that the subscript of $I$ is positive for the first integral in (A.5) and negative for the second one. By symmetry we arrive at the same formula, except that the indicator sets $\left\{N \leq u^{*}\right\}$ and $\left\{N \geq u^{*}\right\}$ are interchanged, which gives (A.2).

\section{A.2 The Laplace transform of $T_{\ell}$ occurring before $d$}

We define $\psi_{\ell}(\lambda ; d)$, the Laplace transform of the distribution of the hitting time of level $\ell$ by standard Brownian motion $W$ restricted to the set where this hitting time occurs before $d$, by

$$
\psi_{\ell}(\lambda ; d):=\mathbb{E}\left[e^{-\lambda T_{\ell}} 1_{\left\{T_{\ell} \leq d\right\}}\right], \quad \lambda \geq 0
$$


Now we construct a stopping time $H=T_{\ell} \wedge d$ and use the martingale $M_{t}=e^{-\frac{z^{2}}{2} t+z W_{t}}$ for our computation. As $H$ is bounded, we can use optional sampling to arrive at

$$
1=\mathbb{E}\left[M_{H}\right]=e^{z \ell} \mathbb{E}\left[e^{-\frac{z^{2}}{2} T_{\ell}} 1_{\left\{T_{\ell} \leq d\right\}}\right]+e^{-\frac{z^{2}}{2} d} \mathbb{E}\left[e^{z W_{d}} 1_{\left\{T_{\ell}>d\right\}}\right]
$$

The second expectation on the right-hand side can be explicitly calculated as the density $\mathbb{P}\left[W_{d} \in d x ; T_{\ell}>d\right]$ is well known (see e.g. [9]). We obtain

$$
\begin{aligned}
\mathbb{E}\left[e^{z W_{d}} 1_{\left\{T_{\ell}>d\right\}}\right] & =\frac{1}{\sqrt{2 \pi d}} \int_{-\infty}^{b} e^{z x}\left(e^{-\frac{x^{2}}{2 d}}-e^{-\frac{(x-2 \ell)^{2}}{2 d}}\right) \mathrm{d} x \\
& =e^{\frac{z^{2} d}{2}}\left[\mathcal{N}\left(\frac{\ell-z d}{\sqrt{d}}\right)-e^{2 \ell z} \mathcal{N}\left(\frac{-\ell-z d}{\sqrt{d}}\right)\right] .
\end{aligned}
$$

Here we assumed $\ell \geq 0$. The calculations for $\ell \leq 0$ proceed in the same way and we can write a general result for all $\ell$ and $\lambda \geq 0$ by

$$
\psi_{\ell}(\lambda ; d)=e^{-|\ell| \sqrt{2 \lambda}} \mathcal{N}\left(\frac{-|\ell|+\sqrt{2 \lambda} d}{\sqrt{d}}\right)+e^{|\ell| \sqrt{2 \lambda}} \mathcal{N}\left(\frac{-|\ell|-\sqrt{2 \lambda} d}{\sqrt{d}}\right) .
$$

\section{A.3 UI property of $e^{-\frac{1}{2} \lambda^{2}(t \wedge \tau)+\lambda W_{t \wedge \tau}}$}

In order to prove the UI property we need the following two lemmas, where the first is on the distribution of stopped Brownian motion.

Lemma A.2 Let $\tau^{+}$be the Parisian stopping time of the upper level. Then for any $t>0$ and $x \geq \ell_{2}$,

$$
\mathbb{P}\left[W_{t \wedge \tau^{+}}>x\right] \leq \mathbb{P}\left[W_{\tau^{+}}>x\right]
$$

Proof First, recall that $W_{\tau^{+}}-\ell_{2}$ is distributed as $\sqrt{D_{2}} N$ and by (3.1) we can compute the probability on the right-hand side as

$$
\mathbb{P}\left[W_{\tau^{+}} \geq x\right]=\int_{\frac{x-\ell_{2}}{\sqrt{D_{2}}}}^{\infty} y e^{-\frac{y^{2}}{2}} \mathrm{~d} y=e^{-\frac{\left(x-\ell_{2}\right)^{2}}{2 D_{2}}} .
$$

Now rewrite the left-hand side probability in (A.7) by

$$
\mathbb{P}\left[W_{t \wedge \tau^{+}}>x\right]=\mathbb{P}\left[W_{\tau^{+}}>x \mid t \geq \tau^{+}\right] \mathbb{P}\left[t \geq \tau^{+}\right]+\mathbb{P}\left[W_{t}>x \mid t<\tau^{+}\right] \mathbb{P}\left[t<\tau^{+}\right],
$$

so we have to find a bound for the second conditional probability on the right-hand side. Assume that $t \geq D_{2}$ and let for $s \geq 0$ the random time $T_{\ell_{2}}^{(s)}$ be given by

$$
T_{\ell_{2}}^{(s)}=\inf \left\{t \geq s \mid W_{t}=\ell_{2}\right\},
$$


denoting the first time that Brownian motion hits the level $\ell_{2}$ after time $s$. We can write for the conditional probability

$$
\begin{aligned}
\mathbb{P}\left[W_{t}>x \mid t<\tau^{+}\right]= & \mathbb{P}\left[W_{t}>x \mid \tau^{+} \notin\left[0, t-D_{2}\right) \cap T_{\ell_{2}}^{\left(t-D_{2}\right)} \in\left[t-D_{2}, t\right)\right] \\
= & \mathbb{P}\left[W_{t}>x \mid T_{\ell_{2}}^{\left(t-D_{2}\right)} \in\left[t-D_{2}, t\right)\right] \\
= & \int_{t-D_{2}}^{t} \mathbb{P}\left[W_{t-\tilde{t}}>x-\ell_{2}\right] \\
& \times \mathbb{P}\left[T_{\ell_{2}}^{\left(t-D_{2}\right)} \in \mathrm{d} \tilde{t} \mid T_{\ell_{2}}^{\left(t-D_{2}\right)} \in\left[t-D_{2}, t\right)\right] \\
\leq & \mathbb{P}\left[W_{D_{2}}>x-\ell_{2}\right],
\end{aligned}
$$

where we used the strong Markov property to restart the process after $T_{\ell_{2}}^{(s)}$. Now we have to show that the probability in (A.9) is smaller than that in (A.8), so we compute

$$
\begin{aligned}
\mathbb{P}\left[W_{D_{2}}>x-\ell_{2}\right] & =\frac{1}{\sqrt{2 \pi D_{2}}} \int_{x}^{\infty} e^{-\frac{\left(y-\ell_{2}\right)^{2}}{2 D_{2}}} \mathrm{~d} y \\
& =\frac{e^{-\frac{\left(x-\ell_{2}\right)^{2}}{2 D_{2}}}}{\sqrt{2 \pi D_{2}}} \int_{x}^{\infty} e^{-\frac{\left(y-\ell_{2}\right)^{2}-\left(x-\ell_{2}\right)^{2}}{2 D_{2}}} \mathrm{~d} y \\
{[3 p t] } & \leq \frac{e^{-\frac{\left(x-\ell_{2}\right)^{2}}{2 D_{2}}}}{\sqrt{2 \pi D_{2}}} \int_{x}^{\infty} e^{-\frac{(y-x)^{2}}{2 D_{2}}} \mathrm{~d} y=\frac{1}{2} \mathbb{P}\left[W_{\tau^{+}} \geq x\right] .
\end{aligned}
$$

For $t<D_{2}$, we have that

$$
\mathbb{P}\left[W_{t}>x \mid t<\tau^{+}\right]=\mathbb{P}\left[W_{t}>x\right] \leq \mathbb{P}\left[W_{D_{2}}>x\right] \leq \mathbb{P}\left[W_{D_{2}}>x-\ell_{2}\right]
$$

For the last probability we have already shown that it satisfies the necessary inequality to prove the lemma.

The following lemma relates the inequality of the distribution of the previous lemma to an inequality of the expectations.

Lemma A.3 Let $X$ and $Y$ be random variables such that we have for every $x>x_{0}$ the inequality

$$
\mathbb{P}[X>x] \leq \mathbb{P}[Y>x] .
$$

Then for any $\ell>x_{0}$, we have for the expectations the inequality

$$
\mathbb{E}\left[X 1_{\{X>\ell\}}\right] \leq \mathbb{E}\left[Y 1_{\{Y>\ell\}}\right]
$$


Proof Let $h^{(n)}=2^{-n}$; then the result follows from the approximation

$$
\begin{aligned}
\mathbb{E}\left[X 1_{\{X>\ell\}}\right] & =\lim _{n \rightarrow \infty} \mathbb{E}\left[\ell 1_{\{X>\ell\}}+h^{(n)} \sum_{k=1}^{\infty} 1_{\left\{X>\ell+k h^{(n)}\right\}}\right] \\
& \leq \lim _{n \rightarrow \infty} \mathbb{E}\left[\ell 1_{\{Y>\ell\}}+h^{(n)} \sum_{k=1}^{\infty} 1_{\left\{Y>\ell+k h^{(n)}\right\}}\right]=\mathbb{E}\left[Y 1_{\{Y>\ell\}}\right],
\end{aligned}
$$

where we get from the first to the second line by using (A.10).

Now we have the tools to prove the UI property of the stopped martingale $\left(M_{t}^{\tau}\right)_{t \geq 0}$ given by

$$
M_{t}^{\tau}=e^{-\frac{1}{2} \lambda^{2}(t \wedge \tau)+\lambda W_{t \wedge \tau}} .
$$

Consider $\lambda>0$. Then we have

$$
M_{t}^{\tau} \leq e^{\lambda W_{t \wedge \tau}} \quad \text { a.s. }
$$

so it remains to show that $e^{\lambda W_{t \wedge \tau}}$ is UI. By Lemma A.2 we have for $x \geq e^{\lambda \ell_{2}}$ the inequality

$$
\mathbb{P}\left[e^{\lambda W_{\tau \wedge t}}>x\right] \leq \mathbb{P}\left[e^{\lambda W_{\tau^{+}} \wedge t}>x\right] \leq \mathbb{P}\left[e^{\lambda W_{\tau^{+}}}>x\right],
$$

which results by Lemma A.3 for $H>e^{\lambda \ell_{2}}$ and $h=\lambda^{-1} \ln (H)$ in

$$
\mathbb{E}\left[e^{\lambda W_{t \wedge \tau}} 1_{\left\{e^{\lambda W_{t \wedge \tau}}>H\right\}}\right] \leq \mathbb{E}\left[e^{\lambda W_{\tau^{+}}} 1_{\left\{W_{\tau^{+}}>h\right\}}\right] .
$$

The distribution of $W_{\tau^{+}}$is known, so we have for $h^{\prime}=D_{2}^{-\frac{1}{2}} h$

$$
\mathbb{E}\left[e^{\lambda W_{\tau^{+}}} 1_{\left\{W_{\tau^{+}}>h\right\}}\right]=e^{\lambda \ell_{2}} \mathbb{E}\left[e^{\lambda \sqrt{D_{2}} N} 1_{\left\{N>h^{\prime}\right\}}\right]=e^{\lambda \ell_{2}} \Psi_{h^{\prime}}\left(\lambda \sqrt{D_{2}}\right) .
$$

The expression on the right-hand side can be made arbitrarily small by increasing $h^{\prime}$, which proves the UI property for $\lambda>0$. For $\lambda<0$ the proof is symmetric, where we have to formulate Lemma A.2 in terms of $\tau_{-}$.

\section{A.4 Discretization error}

If we define the function $f$ by

$$
f(t)=e^{-a t} P_{r}(t) 1_{\{t \geq 0\}},
$$

then the Fourier transform $\phi$ we compute throughout the paper is in fact the Fourier transform of this function $f$. Now define the periodic function $f_{p}$ by

$$
f_{p}(t):=\sum_{j=-\infty}^{\infty} f\left(t+\frac{2 \pi j}{h}\right) .
$$


This sum is uniformly in $t$ bounded by

$$
f_{p}(t) \leq \sum_{j=0}^{\infty} e^{-a \frac{2 \pi j}{h}} \leq \frac{h}{2 \pi} \int_{-\frac{2 \pi}{h}}^{\infty} e^{-a x} \mathrm{~d} x=\frac{h}{2 \pi a} e^{\frac{2 \pi a}{h}} .
$$

Now $f_{p}$ is periodic with period $\frac{2 \pi}{h}$, so we obtain its Fourier series by

$$
f_{p}(t)=\sum_{n=-\infty}^{\infty} \frac{h}{2 \pi} c_{n} e^{i n h t}, \quad \text { where } c_{n}=\int_{-\pi / h}^{\pi / h} f_{p}(t) e^{-i n h t} \mathrm{~d} t .
$$

Calculation of the coefficients $c_{n}$ gives

$$
\begin{aligned}
c_{n} & =\int_{-\pi / h}^{\pi / h} f_{p}(t) e^{-i n h t} \mathrm{~d} t=\int_{-\pi / h}^{\pi / h} \sum_{j=-\infty}^{\infty} f\left(t+\frac{2 \pi j}{h}\right) e^{-i n h t} \mathrm{~d} t \\
& =\sum_{j=-\infty}^{\infty} \int_{-\pi / h}^{\pi / h} f\left(t+\frac{2 \pi j}{h}\right) e^{-i n h t} \mathrm{~d} t \\
& =\int_{-\infty}^{\infty} f(t) e^{-i n h t} \mathrm{~d} t=\phi(-n h) .
\end{aligned}
$$

The interchange of sum and integral is allowed by dominated convergence. Using the coefficients in (A.13) and the equality between (A.12) and (A.11), we obtain the Poisson summation formula

$$
\sum_{j=-\infty}^{\infty} f\left(t+\frac{2 \pi j}{h}\right)=\frac{h}{2 \pi} \sum_{n=-\infty}^{\infty} \phi(-n h) e^{i n h t} .
$$

We can derive the same result for the symmetric function $g$ defined by $g(t)=f(|t|)$. For a fixed $t>0$ we can set $h=\frac{\delta}{t}$ and obtain

$$
\begin{aligned}
\sum_{j=-\infty}^{\infty} g\left(t\left(1+\frac{2 \pi}{\delta} j\right)\right) & =\frac{\delta}{2 \pi t} \sum_{n=-\infty}^{\infty} \phi_{g}(-n h) e^{i n h t} \\
& =\frac{\delta}{\pi t} \Re\{\phi(0)\}+\frac{2 \delta}{\pi t} \sum_{n=1}^{\infty} \Re\left\{\phi\left(\frac{n \delta}{t}\right)\right\} \cos (n \delta) .
\end{aligned}
$$

Using $f(t)=g(t)$ for $t>0$ we have

$$
\begin{aligned}
f(t)=g(t)= & \frac{\delta}{\pi t} \mathfrak{R}\{\phi(0)\}+\frac{2 \delta}{\pi t} \sum_{n=1}^{\infty} \mathfrak{R}\left\{\phi\left(\frac{n \delta}{t}\right)\right\} \cos (n \delta) \\
& -\sum_{|j|>0} g\left(t\left(1+\frac{2 \pi}{\delta} j\right)\right) .
\end{aligned}
$$


In order to get an estimate for the error, we need to control the last sum term. Suppose we choose $\delta<2 \pi$, then we have for this error term $\epsilon$ that

$$
\begin{aligned}
\epsilon & =\sum_{|j|>0} g\left(t\left(1+\frac{2 \pi}{\delta} j\right)\right) \leq \sum_{|j|>0} \exp \left(-a t\left|1+\frac{2 \pi}{\delta} j\right|\right) \\
& \leq 2 \sum_{j=1}^{\infty} \exp \left(-a t\left(\frac{2 \pi}{\delta} j-1\right)\right) \leq \frac{2 e^{-a\left(\frac{2 \pi}{\delta}-1\right) t}}{1-e^{-a \frac{2 \pi}{\delta} t} .}
\end{aligned}
$$

\section{A.5 Truncation error}

If we approximate the integral by a sum, we have

$$
\int_{0}^{\infty} \cos (v T) \Re\{\phi(v)\} \mathrm{d} v \approx h \sum_{j=1}^{\infty} \cos \left(v_{j} T\right) \Re\left\{\phi\left(v_{j}\right)\right\} .
$$

Here $v_{j}=h(j-1)$. To get a bound on the truncation error $\epsilon$, we have to calculate

$$
\epsilon=\left|h \sum_{j=N+1}^{\infty} \cos \left(v_{j} T\right) \Re\left\{\phi\left(v_{j}\right)\right\}\right| \leq h \sum_{j=N+1}^{\infty}\left|\Re\left\{\phi\left(v_{j}\right)\right\}\right|=h \sum_{j=N+1}^{\infty} \epsilon_{j} .
$$

For the $\epsilon_{j}$ we can write

$$
\begin{aligned}
\epsilon_{j} & =\left|\Re \int_{0}^{\infty} e^{i v_{j} t} f(t) \mathrm{d} t\right| \leq\left|\int_{0}^{\infty} \cos \left(v_{j} t\right) e^{-\alpha t} \mathrm{~d} t\right| \\
& \left.=e^{-\alpha t} \frac{v_{j} \sin \left(v_{j} t\right)-\alpha \cos \left(v_{j} t\right)}{\alpha^{2}+v_{j}^{2}}\right]_{t=0}^{t=\infty}=\frac{\alpha}{\alpha^{2}+v_{j}^{2}} .
\end{aligned}
$$

As the term $\frac{\alpha}{\alpha^{2}+v_{j}^{2}}$ is decreasing in $v_{j}$, we can write

$$
\epsilon \leq h \sum_{j=N+1}^{\infty} \frac{\alpha}{\alpha^{2}+v_{j}^{2}} \leq \int_{v_{N}}^{\infty} \frac{\alpha}{\alpha^{2}+v^{2}} \mathrm{~d} v=\arctan \left(\frac{\alpha}{h(N-1)}\right)
$$

\section{A.6 Equation (3.3) for $\tau^{+}$}

In this appendix we start giving a more general form of (3.3). We then use this result to arrive at (3.3) for stopping times.

Lemma A.4 Equation (3.3) can be generalized to the form

$$
\begin{aligned}
\mathbb{E}[ & \left.f\left(n_{t_{n}}^{\ell}\right) g\left(\theta_{t_{1}}^{\ell}, \ldots, \theta_{t_{n}}^{\ell}, \gamma_{t_{1}}^{\ell}, \ldots, \gamma_{t_{n}}^{\ell}\right) 1_{\left\{T_{\ell}<t_{1}\right\}} \mid \mathcal{F}_{T_{\ell}}\right] \\
& =\mathbb{E}[f(N)] \mathbb{E}\left[g\left(\theta_{t_{1}}^{\ell}, \ldots, \theta_{t_{n}}^{\ell}, \gamma_{t_{1}}^{\ell}, \ldots, \gamma_{t_{n}}^{\ell}\right) 1_{\left\{T_{\ell}<t_{1}\right\}} \mid \mathcal{F}_{T_{\ell}}\right],
\end{aligned}
$$

where $\theta_{t}^{\ell}=\operatorname{sgn}\left(W_{t}-\ell\right)$ and $t_{1}<t_{2}<\cdots<t_{n}$. 
Proof The lemma holds because the remark in Sect. 2 that $n_{t}$ is independent of the pair $\left(\gamma_{t}, \operatorname{sgn}\left(W_{t}\right)\right)$ can be extended to the statement that $n_{t}$ is independent of the pair $\left(\gamma_{u}, \operatorname{sgn}\left(W_{u}\right)\right)$ for every $0 \leq u \leq t$. Here we show this extension. Denote by $T_{u}$ the first hitting time of zero after time $u$ and define $\tau=T_{u} \wedge t$. Now we can use the stopping time $\tau$ to write

$$
\begin{aligned}
\mathbb{E}\left[f\left(n_{t}\right) g\left(\theta_{u}, \gamma_{u}\right)\right] & =\mathbb{E}\left[f\left(n_{t}\right) g\left(\theta_{t}, \gamma_{t}\right) 1_{\{\tau=t\}}\right]+\mathbb{E}\left[f\left(n_{t}\right) g\left(\theta_{u}, \gamma_{u}\right) 1_{\{u \leq \tau<t\}}\right] \\
& =\mathbb{E}\left[f\left(n_{t}\right) g\left(\theta_{t}, \gamma_{t}\right) 1_{\left\{\gamma_{t}<u\right\}}\right]+\mathbb{E}\left[f\left(n_{t}\right) g\left(\theta_{u}, \gamma_{u}\right) 1_{\{u \leq \tau<t\}}\right]
\end{aligned}
$$

The first expectation on the right-hand side can by the independence of $n_{t}$ and the pair $\left(\operatorname{sgn}\left(W_{t}\right), \gamma_{t}\right)$ be written as

$$
\begin{aligned}
\mathbb{E}\left[f\left(n_{t}\right) g\left(\gamma_{t}\right) 1_{\left\{\gamma_{t}<u\right\}}\right] & =\mathbb{E}\left[f\left(n_{t}\right)\right] \mathbb{E}\left[g\left(\theta_{t}, \gamma_{t}\right) 1_{\left\{\gamma_{t}<u\right\}}\right] \\
& =\mathbb{E}\left[f\left(n_{t}\right)\right] \mathbb{E}\left[g\left(\theta_{u}, \gamma_{u}\right) 1_{\{\tau=t\}}\right]
\end{aligned}
$$

For the second expectation, we can use the strong Markov property of Brownian motion to get

$$
\begin{aligned}
\mathbb{E} & {\left[f\left(n_{t}\right) g\left(\theta_{u}, \gamma_{u}\right) 1_{\{u \leq \tau<t\}}\right] } \\
& =\mathbb{E}\left[\mathbb{E}\left[f\left(n_{t}\right) g\left(\theta_{u}, \gamma_{u}\right) 1_{\{u \leq \tau<t\}} \mid \mathcal{F}_{\tau}\right]\right] \\
& =\mathbb{E}\left[g\left(\theta_{u}, \gamma_{u}\right) 1_{\{u \leq \tau<t\}} \mathbb{E}\left[f\left(n_{t}\right) \mid \mathcal{F}_{\tau}\right]\right] \\
& =\mathbb{E}\left[g\left(\theta_{u}, \gamma_{u}\right) 1_{\{u \leq \tau<t\}} \mathbb{E}\left[f\left(n_{t-s}\right)\right]_{s=\tau}\right] \\
& =\mathbb{E}\left[f\left(n_{t}\right)\right] \mathbb{E}\left[g\left(\theta_{u}, \gamma_{u}\right) 1_{\{u<\tau \leq t\}}\right] .
\end{aligned}
$$

The substitution of (A.15) and (A.16) into (A.14) shows the extension of the independence result and yields the proof of the lemma.

Lemma A.5 Equation (3.3) also holds for $\tau^{+}$.

Proof Approximate the stopping time $\tau^{+}$by the sequence of stopping times

$$
\tau_{n}=\sum_{k=0}^{\infty} t_{k+1} 1_{\left\{t_{k} \leq \tau^{+}<t_{k+1}\right\}},
$$

where $t_{k}=k / n$. Then we can write (3.3) for the stopping time $\tau_{n}$ as

$$
\begin{aligned}
& \mathbb{E}\left[f\left(n_{\tau_{n}}^{\ell}\right) g\left(\theta_{\tau_{n}}^{\ell}, \gamma_{\tau_{n}}^{\ell}\right) 1_{\left\{T_{\ell}<\tau_{n}\right\}} \mid \mathcal{F}_{T_{\ell}}\right] \\
& \quad=\mathbb{E}\left[\sum_{k=0}^{\infty} f\left(n_{t_{k+1}}^{\ell}\right) g\left(\theta_{t_{k+1}}^{\ell}, \gamma_{t_{k+1}}^{\ell}\right) 1_{\left\{T_{\ell}<t_{k+1}\right\} 1_{\left\{t_{k} \leq \tau^{+}<t_{k+1}\right\}}} \mid \mathcal{F}_{T_{\ell}}\right] .
\end{aligned}
$$


The indicator containing $\tau^{+}$can be rewritten in terms of $\gamma$ and $\theta$ as

$$
\begin{aligned}
\mathbb{E}\left[\sum_{k=0}^{\infty} 1_{\left\{T_{\ell}<t_{k}\right\}} 1_{\left\{t_{k}<\tau_{+} \leq t_{k+1}\right\}} \mid \mathcal{F}_{T_{\ell}}\right] \\
\quad=\mathbb{E}\left[\sum_{k=0}^{\infty} \sum_{j=0}^{k} 1_{\left\{t_{j} \leq T_{\ell}<t_{j+1}\right\}} \prod_{n=j+1}^{k} 1_{\left\{\gamma_{t_{n}}<D\right\} \cup\left\{\theta_{t_{n}}^{\ell}<0\right\}} 1_{\left\{\gamma_{t_{k+1}}>D\right\}} 1_{\left\{\theta_{t_{k+1}}>0\right\}} \mid \mathcal{F}_{T_{\ell}}\right] \\
=\mathbb{E}\left[\sum_{k=0}^{\infty} \sum_{j=0}^{k} 1_{\left\{t_{j} \leq T_{\ell}<t_{j+1}\right\}} h\left(\gamma_{t_{j+1}}, \gamma_{t_{j+2}}, \ldots, \gamma_{t_{k+1}}, \theta_{t_{j+1}}, \theta_{t_{j+2}}, \ldots, \theta_{t_{k+1}}\right) \mid \mathcal{F}_{T_{\ell}}\right] .
\end{aligned}
$$

Now we get the sums out of the expectation. By the previous lemma we get (2.3) for $\tau^{+}$.

\section{References}

1. Abate, J., Valkó, P.: Multi-precision Laplace transform inversion. Int. J. Numer. Methods Eng. 60, 979-993 (2004)

2. Abate, J., Whitt, W.: Numerical inversion of Laplace transforms of probability distributions. ORSA J. Comput. 7, 36-43 (1995)

3. Bernard, C., Le Courtois, O., Quittard-Pinon, F.: A new procedure for pricing Parisian options. J. Deriv. 12(4), 45-53 (2005)

4. Björk, T.: Arbitrage Theory in Continuous Time. Oxford University Press, Oxford (1998)

5. Carr, P., Madan, D.: Option valuation using the fast Fourier transform. J. Comput. Finance 2, 61-79 (1999)

6. Chen, A., Suchanecki, M.: Default risk, bankruptcy procedures and the market value of life insurance liabilities. Insur. Math. Econ. 40, 231-255 (2007)

7. Chesney, M., Gauthier, L.: American Parisian options. Finance Stoch. 10, 475-506 (2006)

8. Chesney, M., Jeanblanc-Picqué, M., Yor, M.: Brownian excursions and Parisian barrier options. Adv. Appl. Probab. 29, 165-184 (1997)

9. Chung, K.L.: Excursions in Brownian motion. Adv. Appl. Probab. 14, 155-177 (1976)

10. Dassios, A., Wu, S.: Two-side Parisian option with single barrier. Preprint, London School of Economics (2008). Available at http://personal.lse.ac.uk/wus2/

11. Gauthier, L.: Excursions height- and length-related stopping times and application to finance. Adv. Appl. Probab. 34, 846-868 (2002)

12. Geman, H., Yor, M.: Pricing and hedging double-barrier options: a probabilistic approach. Math. Finance 6, 365-378 (1996)

13. Haber, R., Schönbucher, P.J., Wilmott, P.: Pricing Parisian options. J. Deriv. 16, 71-79 (1999)

14. Den Iseger, P.: Numerical transform inversion. Probab. Eng. Inf. Sci. 20, 1-44 (2006)

15. Karatzas, I., Shreve, S.: Brownian Motion and Stochastic Calculus, 2nd edn. Springer, Berlin (1991)

16. Kolkiewicz, A.W.: Pricing and hedging more general double-barrier options. J. Comput. Finance 5(3), 1-26 (2002)

17. Labart, C., Lelong, J.: Pricing double-barrier Parisian options using Laplace transforms. CERMICS technical report (2006). http://cermics.enpc.fr/reports/CERMICS-2006/CERMICS-2006-328.pdf

18. Lau, K.W., Kwok, Y.K.: Optimal calling policies in convertible bonds. In: Proceedings of International Conference on Computational Intelligence for Financial Engineering, pp. 109-114. IEEE Press, New York (2003)

19. Moraux, F.: Valuing corporate liabilities when the default threshold is not an absorbing barrier. Working paper, Université de Rennes (2007). Available at http://ssrn.com/abstract=314404

20. Pelsser, A.: Pricing double-barrier options using Laplace transforms. Finance Stoch. 4, 95-104 (2000)

21. Scheffer, C.: The rank of the present excursion. Stoch. Process. Appl. 55, 101-118 (1995)

22. Schröder, M.: Brownian excursions and Parisian barrier options: a note. J. Appl. Probab. 40, 855-864 (2003) 